Momiyama, Kanan, Liu page S1

\title{
Synthesis of Acyclic $\alpha, \beta$-Unsaturated Ketones via Pd(II)-Catalyzed Intermolecular Reaction of Alkynamides and Alkenes
}

\author{
Norie Momiyama, Matthew W. Kanan and David R. Liu* \\ Department of Chemistry \& Chemical Biology, Harvard University \\ Cambridge, Massachusetts 02138
}

\section{Supporting Information}

\section{Experimental Procedures}

General. Reactions were carried out under an ambient atmosphere or standard grade oxygen gas in flame-dried glassware with magnetic stirring. Acetonitrile was dried through alumina. $\mathrm{Na}_{2} \mathrm{PdCl}_{4} \cdot 3 \mathrm{H}_{2} \mathrm{O}$ was purchased from STREM Chemicals. Purification of reaction products was carried out by flash column chromatography using silica gel 60 (E. Merck 230-400 mesh), silica gel 60 silanized (E. Merck), and Florisil ${ }^{\circledR}$ (EM Reagent). Analytical thin layer chromatography (TLC) was performed on E. Merck precoated (0.25 $\mathrm{mm}$ ) silica gel $60-\mathrm{F}_{254}$ plates. Visualization was accomplished with UV light and staining with phosphomolybdic acid solution in ethanol, followed by heating.

${ }^{1} \mathrm{H}$ NMR spectra were recorded on a Varian Inova-500 (500 MHz) at ambient temperature. All NMR solvents were purchased from Cambridge Isotope Laboratories. Data are reported as follows: chemical shift in ppm from a tetramethylsilane internal standard $(\delta)$, multiplicity $(\mathrm{b}=$ broad, $\mathrm{s}=$ singlet, $\mathrm{d}=$ doublet, $\mathrm{t}=$ triplet, $\mathrm{q}=$ quartet, and $\mathrm{m}=$ multiplet), integration, coupling constants where appropriate $(\mathrm{Hz})$, and assignment. ${ }^{13} \mathrm{C}$ NMR was recorded on a Varian Inova-500 (125 MHz) at ambient temperature, with the resonance of deuterochloroform at $77.0 \mathrm{ppm}$ used as an internal standard. Note that amide rotamers

General Procedure for Synthesis of Pentyn- or Hexynamide. To a solution of corresponding amine $(2.0 \mathrm{mmol}, 1$ equiv) in $\mathrm{MeCN}(10 \mathrm{~mL})$ was added $N$ Hydroxysuccinimide-activated 4-Pentynoic or 5-Hexynoic acid (2.4 mmol, 1.2 equiv) at room temperature. After stirring at room temperature for $1 \mathrm{~h}$, the resulting solution was treated with saturated aqueous $\mathrm{NH}_{4} \mathrm{Cl}$. The aqueous layer was extracted with EtOAc (20 $\mathrm{mL} \times 2$ ) and the combined organic layer was washed with brine (10 $\mathrm{mL} \times 2)$ and dried over $\mathrm{Na}_{2} \mathrm{SO}_{4}$. After filtration followed by evaporation, the crude product was purified by flash column chromatography on silica gel to provide the title compounds.

General Procedure for Intermolecular Reaction of Alkynamides and Alkenes. To a solution of $\mathrm{Na}_{2} \mathrm{PdCl}_{4} \cdot 3 \mathrm{H}_{2} \mathrm{O}(15.7 \mathrm{mg}, 0.045 \mathrm{mmol}, 15 \mathrm{~mol} \%)$ and $\mathrm{CuCl}_{2} \cdot 2 \mathrm{H}_{2} \mathrm{O}(10.2 \mathrm{mg}$, $0.060 \mathrm{mmol}, 20 \mathrm{~mol} \%)$ in $\mathrm{MeCN}: \mathrm{H}_{2} \mathrm{O} 5: 1(1.5 \mathrm{~mL})$ was added alkene $(0.45 \mathrm{mmol}, 1.5$ eq). The reaction vessel was capped and placed under $1 \mathrm{~atm}$ of oxygen. A solution of alkyne $(0.30 \mathrm{mmol}, 1.0 \mathrm{eq})$ in $\mathrm{MeCN}: \mathrm{H}_{2} \mathrm{O} 5: 1(1.5 \mathrm{~mL})$ was added dropwise over 8-12 h at room temperature or $40{ }^{\circ} \mathrm{C}$. After the dropwise addition was completed, the reaction mixture was poured into saturated aqueous $\mathrm{NH}_{4} \mathrm{Cl}(5 \mathrm{~mL})$. The aqueous layer was extracted with EtOAc $(5 \mathrm{~mL} \times 3$ ), and then the organic layer was washed with brine (5 $\mathrm{mL} \times 2$ ). The combined organic layers were dried over $\mathrm{Na}_{2} \mathrm{SO}_{4}$ and filtered, and the solvent was removed by rotary evaporation. The crude product was purified by flash 
chromatography with a mobile phase consisting of a mixture of hexanes and ethyl acetate and a solid phase consisting of a layer of Florisil on top of a layer of silica gel. In all cases below, the $E$ isomer was the only isomer observed by NMR analysis. Note that amide isomers in some cases cause the appearance of two very closely spaced resonances in both protein and carbon NMR spectra.

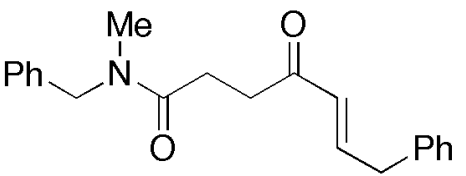

(E)- $N$-Benzyl- $N$-methyl-4-oxo-7-phenylhept-5-enamide. TLC $\mathrm{R}_{f}=0.15$ (2:1 hexane: ethyl acetate); ${ }^{1} \mathrm{H} \mathrm{NMR}\left(\mathrm{CDCl}_{3}\right.$, $500 \mathrm{MHz}) \delta 7.17-7.38(\mathrm{~m}, 10 \mathrm{H}, \mathrm{Ar}-H), 7.01-7.07(\mathrm{~m}, 1 \mathrm{H}$, $\mathrm{CH}), 6.13,6.15(\mathrm{dt}, 1 \mathrm{H}, J=15.5,1.5 \mathrm{~Hz}, \mathrm{CH}), 4.58,4.59$ (s, $\left.2 \mathrm{H}, \mathrm{CH}_{2}\right), 3.55\left(\mathrm{t}, \mathrm{J}=5.0 \mathrm{~Hz}, 2 \mathrm{H}, \mathrm{CH}_{2}\right), 2.93,2.96\left(\mathrm{~s}, 3 \mathrm{H}, \mathrm{CH}_{3}\right), 2.92-2.99\left(\mathrm{~m}, 2 \mathrm{H}, \mathrm{CH}_{2}\right)$, 2.68-2.71 (m, 2H, $\left.\mathrm{CH}_{2}\right) ;{ }^{13} \mathrm{C} \mathrm{NMR}\left(100 \mathrm{MHz}, \mathrm{CDCl}_{3}\right) \delta 199.1(\mathrm{C}=\mathrm{O}), 199.2(\mathrm{C}=\mathrm{O}), 171.8$ $(\mathrm{NC}=\mathrm{O}), 172.0(\mathrm{NC}=\mathrm{O}), 145.5(\mathrm{CH}), 145.6(\mathrm{CH}), 137.3,136.5,130.9(\mathrm{CH}), 131.1(C \mathrm{H})$, 128.9, 128.8 (2C), 128.7 (2C), 128.5, 127.9, 127.5, 127.2, 126.7, 126.4, 53.2, 50.9, 38.9, 38.7, 34.9, 34.8, 33.9, 27.1, 26.8; MS (ESI) Exact mass calculated for $\mathrm{C}_{21} \mathrm{H}_{23} \mathrm{NO}_{2}$ $(\mathrm{M}+\mathrm{H})^{+}: 322.1729$. Found: 322.1663 .

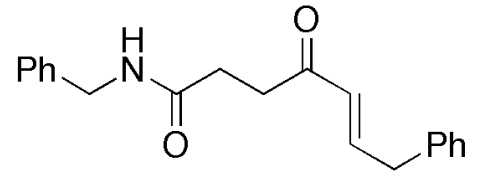

(E)-N-Benzyl-4-oxo-7-phenylhept-5-enamide. TLC $\mathrm{R}_{f}=$ 0.26 (1:1 hexane: ethyl acetate); ${ }^{1} \mathrm{H}$ NMR $\left(\mathrm{CDCl}_{3}, 500\right.$ $\mathrm{MHz})$ 87.29-7.33 (m, 4H, Ar- $H$ ), 7.25-7.26 (m, 4H, Ar-H), 7.16-7.17 (d, 2H, $J=6.5 \mathrm{~Hz}, \operatorname{Ar}-H), 6.99(\mathrm{dt}, 1 \mathrm{H}, J=16.0$, $6.5 \mathrm{~Hz}, \mathrm{CH}), 6.10(\mathrm{~d}, 1 \mathrm{H}, J=15.5 \mathrm{~Hz}), 6.03(\mathrm{br}, 1 \mathrm{H}, \mathrm{NH}), 4.41\left(\mathrm{~d}, 2 \mathrm{H}, J=6.0 \mathrm{~Hz}, \mathrm{CH}_{2}\right)$, $3.54\left(\mathrm{~d}, 2 \mathrm{H}, J=6.5 \mathrm{~Hz}, \mathrm{CH}_{2}\right), 2.95\left(\mathrm{t}, 2 \mathrm{H}, J=7.0 \mathrm{~Hz}, \mathrm{CH}_{2}\right), 2.50(\mathrm{t}, 2 \mathrm{H}, J=7.0 \mathrm{~Hz}$, $\left.\mathrm{CH}_{2}\right) ;{ }^{13} \mathrm{C}$ NMR $\left(100 \mathrm{MHz}, \mathrm{CDCl}_{3}\right) \delta$ 199.3, 171.9, 146.1, 138.2, 137.5, 130.7, 128.8 (2C), 128.7 (2C), 128.6 (2C), 127.7 (2C), 127.4, 126.7, 43.6, 38.7, 35.2, 29.9; MS (ESI) Exact mass calculated for $\mathrm{C}_{20} \mathrm{H}_{21} \mathrm{NO}_{2}(\mathrm{M}+\mathrm{H})^{+}: 308.1572$. Found: 308.1519 .

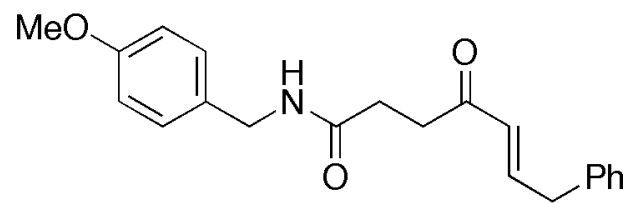

(E)-N-(4-methoxybenzyl)-4-oxo-7-phenylhept5-enamide. TLC $\mathrm{R}_{f}=0.25$ (1:1 hexane: ethyl acetate); ${ }^{1} \mathrm{H} \mathrm{NMR}\left(\mathrm{CDCl}_{3}, 500 \mathrm{MHz}\right) \delta 7.32(\mathrm{t}, 2 \mathrm{H}$, $J=7.5 \mathrm{~Hz}, \operatorname{Ar}-H), 7.25(\mathrm{t}, 1 \mathrm{H}, J=7.5 \mathrm{~Hz}, \operatorname{Ar}-H)$, $7.19(\mathrm{~d}, 2 \mathrm{H}, J=9.0 \mathrm{~Hz}), 7.16(\mathrm{~d}, 2 \mathrm{H}, J=8.0 \mathrm{~Hz}$, Ar-H), $7.00(\mathrm{dt}, 1 \mathrm{H}, J=15.5,7.0 \mathrm{~Hz}, \mathrm{CH}), 6.85$ (d, 2H, $J=9.0 \mathrm{~Hz}, \mathrm{Ar}-H), 6.10$ (dt, $1 \mathrm{H}, J$ $=15.5,1.5 \mathrm{~Hz}, \mathrm{CH}), 5.98(\mathrm{br}, 1 \mathrm{H}, \mathrm{NH}), 4.34\left(\mathrm{~d}, 2 \mathrm{H}, J=6.0 \mathrm{~Hz}, \mathrm{CH}_{2}\right), 3.78(\mathrm{~s}, 3 \mathrm{H}$, $\left.\mathrm{OCH}_{3}\right), 3.54\left(\mathrm{~d}, 2 \mathrm{H}, J=7.0 \mathrm{~Hz}, \mathrm{CH}_{2}\right), 2.94\left(\mathrm{t}, 2 \mathrm{H}, J=6.5 \mathrm{~Hz}, \mathrm{CH}_{2}\right), 2.48(\mathrm{t}, 2 \mathrm{H}, J=6.5$ $\left.\mathrm{Hz}, \mathrm{CH}_{2}\right) ;{ }^{13} \mathrm{C} \mathrm{NMR}\left(100 \mathrm{MHz}, \mathrm{CDCl}_{3}\right) \delta 199.0,171.7,158.9,146.0,137.5,130.7,130.3$, 129.0 (2C), 128.8 (2C), 128.7 (2C), 126.7, 114.0 (2C), 55.2, 43.1, 38.7, 35.2, 30.0; MS (ESI) Exact mass calculated for $\mathrm{C}_{21} \mathrm{H}_{23} \mathrm{NO}_{3}(\mathrm{M}+\mathrm{H})^{+}: 338.1678$. Found: 338.1638 .

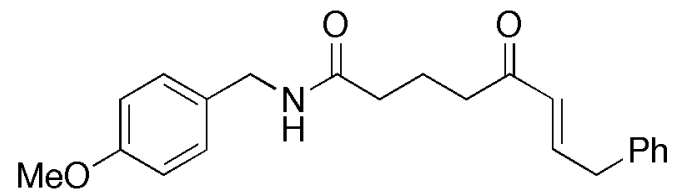

(E)-N-(4-methoxybenzyl)-5-oxo-8-phenyloct6-enamide. TLC $\mathrm{R}_{f}=0.18$ (1:1 hexane: ethyl acetate); ${ }^{1} \mathrm{H}$ NMR $\left(\mathrm{CDCl}_{3}, 500 \mathrm{MHz}\right) \delta 7.32(\mathrm{t}$, $2 \mathrm{H}, J=7.5 \mathrm{~Hz}, \operatorname{Ar}-H), 7.25(\mathrm{t}, 1 \mathrm{H}, J=7.0 \mathrm{~Hz}$, $\operatorname{Ar}-H), 7.19(\mathrm{~d}, 2 \mathrm{H}, \operatorname{Ar}-H, J=9.0 \mathrm{~Hz}), 7.16(\mathrm{~d}, 2 \mathrm{H}, \operatorname{Ar}-H, J=8.0 \mathrm{~Hz}), 6.95(\mathrm{dt}, 1 \mathrm{H}, J=$ 
$15.5,7.0 \mathrm{~Hz}, \mathrm{CH}), 6.84(\mathrm{~d}, 2 \mathrm{H}, J=8.5 \mathrm{~Hz}, \mathrm{Ar}-H), 6.06(\mathrm{~d}, 1 \mathrm{H}, J=16.0 \mathrm{~Hz}, \mathrm{CH}), 5.78(\mathrm{br}$, $1 \mathrm{H}, \mathrm{NH}), 4.34\left(\mathrm{~d}, 2 \mathrm{H}, J=5.5 \mathrm{~Hz}, \mathrm{CH}_{2}\right), 3.78\left(\mathrm{~s}, 3 \mathrm{H}, \mathrm{OCH}_{3}\right), 3.52(\mathrm{~d}, 2 \mathrm{H}, J=7.0 \mathrm{~Hz}$, $\left.\mathrm{CH}_{2}\right), 2.62\left(\mathrm{t}, 2 \mathrm{H}, J=7.0 \mathrm{~Hz}, \mathrm{CH}_{2}\right), 2.22\left(\mathrm{t}, 2 \mathrm{H}, J=7.5 \mathrm{~Hz}, \mathrm{CH}_{2}\right), 1.92-1.98(\mathrm{~m}, 2 \mathrm{H}$, $\left.\mathrm{CH}_{2}\right) ;{ }^{13} \mathrm{C}$ NMR $\left(100 \mathrm{MHz}, \mathrm{CDCl}_{3}\right) \delta 199.9,172.1,158.9,145.6,137.5,130.9,130.4$, 129.1 (2C), 128.7 (2C), 128.6 (2C), 126.7, 114.0 (2C), 55.2, 42.9, 38.7, 38.6, 35.4, 19.9; MS (ESI) Exact mass calculated for $\mathrm{C}_{22} \mathrm{H}_{25} \mathrm{NO}_{3}(\mathrm{M}+\mathrm{H})^{+}: 352.1834$. Found: 352.1852 .

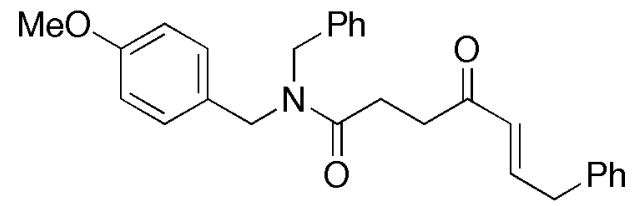

(E)-N-Benzyl- $N$-(4-methoxybenzyl)-4-oxo-7phenyloct-5-enamide. TLC $\mathrm{R}_{f}=0.16 \quad(2: 1$ hexane: ethyl acetate); ${ }^{1} \mathrm{H}$ NMR $\left(\mathrm{CDCl}_{3}, 500\right.$ MHz) $\delta 7.11-7.37(\mathrm{~m}, 10 \mathrm{H}$, Ar- $H)$, 7.00-7.06 (m, $1 \mathrm{H}, \mathrm{CH}), 6.88(\mathrm{~d}, 1 \mathrm{H}, J=8.0 \mathrm{~Hz}, \operatorname{Ar} H), 6.82(\mathrm{~d}$, $1 \mathrm{H}, J=8.0 \mathrm{~Hz}, \operatorname{Ar} H), 6.84(\mathrm{~d}, 2 \mathrm{H}, J=8.5 \mathrm{~Hz}, \mathrm{Ar}-H), 6.14(\mathrm{dt}, 1 \mathrm{H}, J=16.0,1.5 \mathrm{~Hz}, \mathrm{CH})$, $4.55,4.51,4.47,4.43\left(\mathrm{~s}, 4 \mathrm{H}, \mathrm{CH}_{2}\right), 3.80,3.78\left(\mathrm{~s}, 3 \mathrm{H}, \mathrm{OCH}_{3}\right), 3.54(\mathrm{~d}, 2 \mathrm{H}, J=7.0 \mathrm{~Hz}$, $\left.\mathrm{CH}_{2}\right), 2.97\left(\mathrm{q}, 2 \mathrm{H}, J=7.5 \mathrm{~Hz}, \mathrm{CH}_{2}\right), 2.75\left(\mathrm{t}, 1 \mathrm{H}, J=6.5 \mathrm{~Hz}, \mathrm{CH}_{2}\right), 2.71(\mathrm{t}, 1 \mathrm{H}, J=6.5 \mathrm{~Hz}$, $\left.\mathrm{CH}_{2}\right) ;{ }^{13} \mathrm{C}$ NMR $\left(100 \mathrm{MHz}, \mathrm{CDCl}_{3}\right) \delta$ 199.1, 172.1, 158.9, 145.6, 137.6, 137.3, 136.4, 130.9, $129.6(2 \mathrm{C}), 129.3,128.8$ (2C), 128.7 (2C), 128.6 (2C), 128.1, 127.9, 127.5, 127.3, 126.7 (2C), 126.5 (2C) 114.3 (2C), 113.9 (2C), 55.2, 49.6, 49.2, 48.0,47.0, 38.8, 34.8, 27.0; MS (ESI) Exact mass calculated for $\mathrm{C}_{28} \mathrm{H}_{29} \mathrm{NO}_{3}(\mathrm{M}+\mathrm{H})^{+}$: 428.2147. Found: 428.2087.

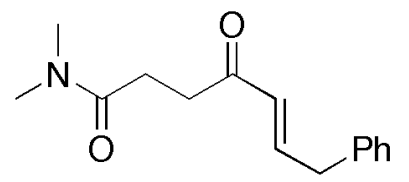

(E)- $\mathrm{N}, \mathrm{N}$-Dimethyl-4-oxo-7-phenylhept-5-enamide. TLC $\mathrm{R}_{f}=$ 0.15 (1:2 hexane: ethyl acetate); ${ }^{1} \mathrm{H}$ NMR $\left(\mathrm{CDCl}_{3}, 500 \mathrm{MHz}\right)$ $\delta 7.32(\mathrm{t}, 2 \mathrm{H}, J=7.5 \mathrm{~Hz}, \mathrm{Ar}-H), 7.24(\mathrm{t}, 1 \mathrm{H}, J=7.5 \mathrm{~Hz}, \mathrm{Ar}-H)$, $7.18(\mathrm{~d}, 2 \mathrm{H}, J=7.0 \mathrm{~Hz}, \mathrm{Ar}-H), 7.02(\mathrm{dt}, 1 \mathrm{H}, J=16.0,6.5 \mathrm{~Hz}$, $\mathrm{CH}), 6.14(\mathrm{dt}, 1 \mathrm{H}, J=16.0,1.5 \mathrm{~Hz}, \mathrm{CH}), 3.54\left(\mathrm{dd}, 2 \mathrm{H}, \mathrm{CH}_{2}, J=5.5,1.5 \mathrm{~Hz}\right), 3.04(\mathrm{~s}, 3 \mathrm{H}$, $\left.\mathrm{CH}_{3}\right), 2.93\left(\mathrm{~s}, 3 \mathrm{H}, \mathrm{CH}_{3}\right), 2.91\left(\mathrm{t}, 2 \mathrm{H}, J=6.5 \mathrm{~Hz}, \mathrm{CH}_{2}\right), 2.63\left(\mathrm{t}, 2 \mathrm{H}, J=7.0 \mathrm{~Hz}, \mathrm{CH}_{2}\right),{ }^{13} \mathrm{C}$ NMR $\left(100 \mathrm{MHz}, \mathrm{CDCl}_{3}\right) \delta 199.3,171.6,145.6,137.6,131.0,128.8$ (2C), 128.7 (2C), 126.7, 38.8, 37.1, 35.5, 34.8, 27.0; MS (ESI) Exact mass calculated for $\mathrm{C}_{15} \mathrm{H}_{19} \mathrm{NO}_{2}$ $(\mathrm{M}+\mathrm{H})^{+}:$246.1416. Found: 246.1433 .

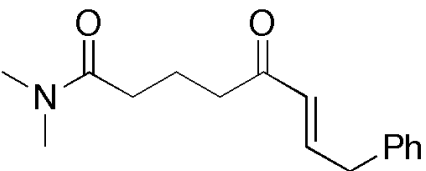

(E)- $N, N$-Dimethyl-5-oxo-8-phenyloct-6-enamide. TLC $\mathrm{R}_{f}=$ 0.18 (1:2 hexane: ethyl acetate); ${ }^{1} \mathrm{H}$ NMR $\left(\mathrm{CDCl}_{3}, 500 \mathrm{MHz}\right)$ $\delta 7.32(\mathrm{t}, 2 \mathrm{H}, J=7.5 \mathrm{~Hz}, \mathrm{Ar}-H), 7.24(\mathrm{t}, 1 \mathrm{H}, J=7.5 \mathrm{~Hz}, \mathrm{Ar}-$ $H), 7.16(\mathrm{~d}, 2 \mathrm{H}, J=7.5 \mathrm{~Hz}, \mathrm{Ar}-H), 6.97(\mathrm{dt}, 1 \mathrm{H}, J=16.0,6.5$ $\mathrm{Hz}, \mathrm{CH}), 6.08(\mathrm{~d}, 1 \mathrm{H}, J=15.5 \mathrm{~Hz}, \mathrm{CH}), 3.53\left(\mathrm{~d}, 2 \mathrm{H}, \mathrm{CH}_{2}, J=6.5 \mathrm{~Hz}\right), 2.98\left(\mathrm{~s}, 3 \mathrm{H}, \mathrm{CH}_{3}\right)$, $2.92\left(\mathrm{~s}, 3 \mathrm{H}, \mathrm{CH}_{3}\right), 2.66$ (t, $\left.2 \mathrm{H}, J=7.0 \mathrm{~Hz}, \mathrm{CH}_{2}\right), 2.34\left(\mathrm{t}, 2 \mathrm{H}, J=7.0 \mathrm{~Hz}, \mathrm{CH}_{2}\right), 1.94$ (t, $2 \mathrm{H}$, $\left.J=7.0 \mathrm{~Hz}, \mathrm{CH}_{2}\right) ;{ }^{13} \mathrm{C}$ NMR $\left(100 \mathrm{MHz}, \mathrm{CDCl}_{3}\right) \delta 200.2,172.4,145.4,137.6,131.0,128.8$ (2C), 128.7 (2C), 126.7, 39.0, 38.7, 37.1, 35.3, 32.2, 19.3; MS (ESI) Exact mass calculated for $\mathrm{C}_{16} \mathrm{H}_{21} \mathrm{NO}_{2}(\mathrm{M}+\mathrm{H})^{+}: 260.1572$. Found: 260.1551.

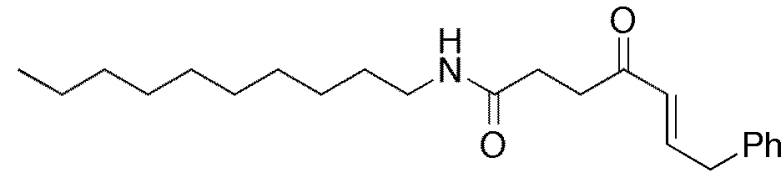

(E)-N-Decyl-4-oxo-7-phenylhept-5enamide. TLC $\mathrm{R}_{f}=0.18$ (2:1 hexane: ethyl acetate); ${ }^{1} \mathrm{H}$ NMR $\left(\mathrm{CDCl}_{3}, 500\right.$ $\mathrm{MHz}) \delta 7.32(\mathrm{t}, 2 \mathrm{H}, J=7.0 \mathrm{~Hz}, \operatorname{Ar}-H)$,

$7.24(\mathrm{t}, 1 \mathrm{H}, J=7.5 \mathrm{~Hz}, \mathrm{Ar}-H), 7.16(\mathrm{~d}, 2 \mathrm{H}, J=7.0 \mathrm{~Hz}, \mathrm{Ar}-H), 6.97$ (dt, $1 \mathrm{H}, J=16.0,6.5$ 
$\mathrm{Hz}, \mathrm{CH}), 6.10(\mathrm{~d}, 1 \mathrm{H}, J=16.0 \mathrm{~Hz}, \mathrm{CH}), 5.77(\mathrm{br}, 1 \mathrm{H}, \mathrm{NH}), 3.53\left(\mathrm{~d}, 2 \mathrm{H}, \mathrm{CH}_{2}, J=6.5 \mathrm{~Hz}\right)$, 3.20 (q, $2 \mathrm{H}, J=6.5 \mathrm{~Hz}, \mathrm{CH}_{2}$ ), $2.92\left(\mathrm{t}, 2 \mathrm{H}, J=6.5 \mathrm{~Hz}, \mathrm{CH}_{2}\right), 2.45\left(\mathrm{t}, 2 \mathrm{H}, J=7.0 \mathrm{~Hz}, \mathrm{CH}_{2}\right.$ ), $1.46\left(\mathrm{br}, 2 \mathrm{H}, \mathrm{CH}_{2}\right), 1.25\left(\mathrm{br}, 14 \mathrm{H}, \mathrm{CH}_{2}\right), 0.88\left(\mathrm{t}, 3 \mathrm{H}, J=7.0 \mathrm{~Hz}, \mathrm{CH}_{3}\right) ;{ }^{13} \mathrm{C}$ NMR $(100$ $\mathrm{MHz}, \mathrm{CDCl}_{3}$ ) $\delta$ 199.2, 171.9, 146.0, 137.5, 130.7, 128.8 (2C), 128.7 (2C), 126.7, 39.6, 38.7, 35.3, 31.8, 30.1, 29.5 (2C), 29.3 (2C), 29.2, 26.8, 22.6, 14.1; MS (ESI) Exact mass calculated for $\mathrm{C}_{23} \mathrm{H}_{35} \mathrm{NO}_{2}(\mathrm{M}+\mathrm{H})^{+}$: 358.2668. Found: 358.2655.

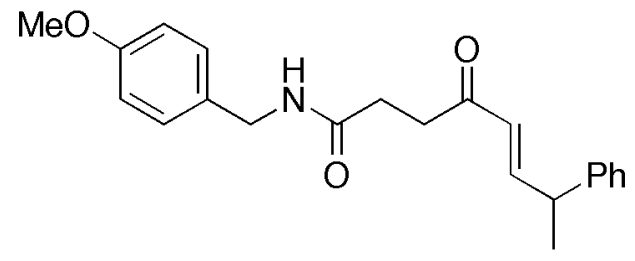

(E)- $N$-(4-methoxybenzyl)-4-oxo-7-phenyoct-5enamide. TLC $\mathrm{R}_{f}=0.28$ (1:1 hexane: ethyl acetate); ${ }^{1} \mathrm{H} \mathrm{NMR}\left(\mathrm{CDCl}_{3}, 500 \mathrm{MHz}\right) \delta 7.31(\mathrm{t}, 2 \mathrm{H}$, $J=7.0 \mathrm{~Hz}, \operatorname{Ar}-H), 7.23(\mathrm{~m}, 1 \mathrm{H}, \operatorname{Ar}-H), 7.17(\mathrm{~d}$, $4 \mathrm{H}, J=7.0 \mathrm{~Hz}, \mathrm{Ar}-H), 7.00(\mathrm{dd}, 1 \mathrm{H}, J=16.2,6.5$ $\mathrm{Hz}, \mathrm{CH}), 6.83(\mathrm{dt}, 2 \mathrm{H}, J=8.0,1.5 \mathrm{~Hz}, \mathrm{Ar}-H)$, $6.08(\mathrm{dd}, 1 \mathrm{H}, J=16.0,2.0 \mathrm{~Hz}, \mathrm{CH}), 5.92(\mathrm{br}, 1 \mathrm{H}, \mathrm{NH}), 4.33\left(\mathrm{~d}, 2 \mathrm{H}, J=5.5 \mathrm{~Hz}, \mathrm{CH}_{2}\right)$, $3.78\left(\mathrm{~s}, 3 \mathrm{H}, \mathrm{OCH}_{3}\right.$ ), 3.61 (quintet, $\left.1 \mathrm{H}, J=7.0 \mathrm{~Hz}, \mathrm{CH}\right), 2.94\left(\mathrm{t}, 2 \mathrm{H}, J=6.5 \mathrm{~Hz}, \mathrm{CH}_{2}\right.$ ), $2.47\left(\mathrm{t}, 2 \mathrm{H}, J=6.5 \mathrm{~Hz}, \mathrm{CH}_{2}\right), 1.42\left(\mathrm{~d}, 3 \mathrm{H}, J=7.0 \mathrm{~Hz}, \mathrm{CH}_{3}\right) ;{ }^{13} \mathrm{C} \mathrm{NMR}\left(100 \mathrm{MHz}, \mathrm{CDCl}_{3}\right)$

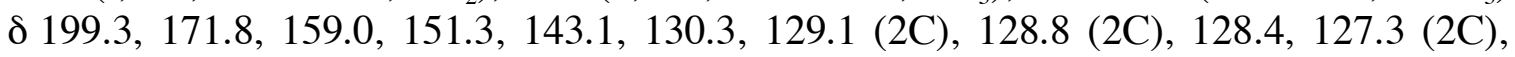
126.8, 114.0 (2C), 55.3, 43.1, 42.3, 35.3, 30.0, 20.1; MS (ESI) Exact mass calculated for $\mathrm{C}_{22} \mathrm{H}_{25} \mathrm{NO}_{3}(\mathrm{M}+\mathrm{H})^{+}:$352.1834. Found: 352.1781 .

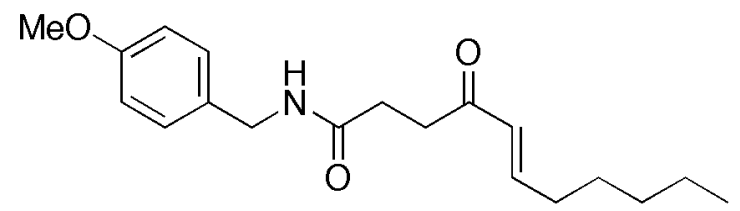

(E)-N-(4-Methoxybenzyl)-4-oxoundec-5enamide. TLC $R_{f}=0.30$ (1:1 hexane: ethyl acetate); ${ }^{1} \mathrm{H} \mathrm{NMR}\left(\mathrm{CDCl}_{3}, 500 \mathrm{MHz}\right) \delta 7.19$ $(\mathrm{d}, 2 \mathrm{H}, J=9.0 \mathrm{~Hz}, \mathrm{Ar}-H), 6.86-6.92(\mathrm{~m}, 1 \mathrm{H}$, $\mathrm{CH}), 6.86(\mathrm{~d}, 2 \mathrm{H}, J=9.0 \mathrm{~Hz}, \mathrm{Ar}-H), 6.11(\mathrm{~d}$, $1 \mathrm{H}, J=14.0 \mathrm{~Hz}, \mathrm{CH}), 5.98$ (brs, $1 \mathrm{H}, \mathrm{NH}), 4.35\left(\mathrm{~d}, 2 \mathrm{H}, J=5.5 \mathrm{~Hz}, \mathrm{CH}_{2}\right), 3.78(\mathrm{~s}, 3 \mathrm{H}$, $\left.\mathrm{OCH}_{3}\right), 2.96\left(\mathrm{t}, 2 \mathrm{H}, J=7.0 \mathrm{~Hz}, \mathrm{CH}_{2}\right), 2.50\left(\mathrm{t}, 2 \mathrm{H}, J=6.5 \mathrm{~Hz}, \mathrm{CH}_{2}\right), 2.21(\mathrm{q}, 2 \mathrm{H}, J=6.0$ $\left.\mathrm{Hz}, \mathrm{CH}_{2}\right), 1.46\left(\mathrm{q}, 2 \mathrm{H}, J=7.0 \mathrm{~Hz}, \mathrm{CH}_{2}\right), 1.29-1.34\left(\mathrm{~m}, 4 \mathrm{H}, \mathrm{CH}_{2}\right), 0.897$ (t, 3H, $J=7.0$ $\left.\mathrm{Hz}, \mathrm{CH}_{3}\right) ;{ }^{13} \mathrm{C} \mathrm{NMR}\left(100 \mathrm{MHz}, \mathrm{CDCl}_{3}\right) \delta 199.2,171.8,158.9,148.4,130.3,130.0,129.0$ (2C), 114.0 (2C), 55.3, 43.1, 35.1, 32.5, 31.3, 30.1, 27.7, 22.4, 13.9; MS (ESI) Exact mass calculated for $\mathrm{C}_{19} \mathrm{H}_{27} \mathrm{NO}_{3}(\mathrm{M}+\mathrm{H})^{+}: 318.1991$. Found: 318.1960 .

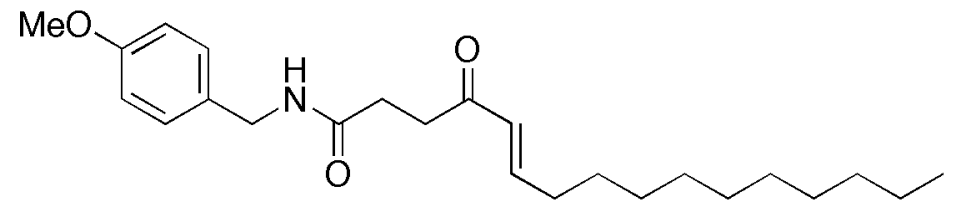

(E)-N-(4-Methoxybenzyl)-4oxohexadec-5-enamide. TLC $\mathrm{R}_{f}=0.20$ (2:1 hexane: ethyl acetate); ${ }^{1} \mathrm{H} \mathrm{NMR}\left(\mathrm{CDCl}_{3}, 500\right.$ $\mathrm{MHz}) \delta 7.19(\mathrm{~d}, 2 \mathrm{H}, J=8.5 \mathrm{~Hz}$,

Ar- $H$ ), 6.84-6.92 (m, 3H, CH, Ar- $H$ ), $6.11(\mathrm{~d}, 1 \mathrm{H}, J=16.0 \mathrm{~Hz}, \mathrm{CH}), 5.96$ (brs, $1 \mathrm{H}, \mathrm{NH})$, $4.35\left(\mathrm{~d}, 2 \mathrm{H}, J=6.0 \mathrm{~Hz}, \mathrm{CH}_{2}\right), 3.79\left(\mathrm{~s}, 3 \mathrm{H}, \mathrm{OCH}_{3}\right), 2.95\left(\mathrm{t}, 2 \mathrm{H}, J=7.0 \mathrm{~Hz}, \mathrm{CH}_{2}\right), 2.50$ (t, $\left.2 \mathrm{H}, J=6.5 \mathrm{~Hz}, \mathrm{CH}_{2}\right), 2.21\left(\mathrm{q}, 2 \mathrm{H}, J=7.0 \mathrm{~Hz}, \mathrm{CH}_{2}\right), 1.44-1.47\left(\mathrm{q}, 2 \mathrm{H}, J=7.0 \mathrm{~Hz}, \mathrm{CH}_{2}\right)$, $1.26\left(\mathrm{br}, 14 \mathrm{H}, \mathrm{CH}_{2}\right), 0.882\left(\mathrm{t}, 3 \mathrm{H}, J=6.5 \mathrm{~Hz}, \mathrm{CH}_{3}\right) ;{ }^{13} \mathrm{C} \mathrm{NMR}\left(100 \mathrm{MHz}, \mathrm{CDCl}_{3}\right) \delta$ 199.2, 171.9, 159.0, 148.4, 130.3, 130.0, 129.0 (2C), 114.0 (2C), 55.3, 43.1, 35.1, 32.5, $32.0,30.3,29.9,29.6,29.5$ (2C), 29.4 (2C), 28.0, 22.7, 14.1; MS (ESI) Exact mass calculated for $\mathrm{C}_{24} \mathrm{H}_{37} \mathrm{NO}_{3}(\mathrm{M}+\mathrm{H})^{+}: 388.2773$. Found: 388.2726. 
$\mathrm{MeO}$

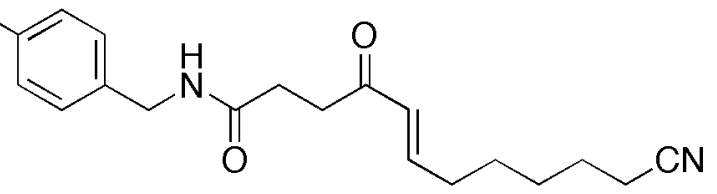

(E)-11-Cyano- $N$-(4-methoxybenzyl)4-oxoundec-5-enamide. TLC $\mathrm{R}_{f}=0.18$ (40:1 $\left.\quad \mathrm{CH}_{2} \mathrm{Cl}_{2}: \mathrm{MeOH}\right) ;{ }^{1} \mathrm{H} \quad \mathrm{NMR}$ $\left(\mathrm{CDCl}_{3}, 500 \mathrm{MHz}\right) \delta 7.21(\mathrm{~d}, 2 \mathrm{H}, J=$ $9.0 \mathrm{~Hz}, \mathrm{Ar}-\mathrm{H}), 6.83-6.89(\mathrm{~m}, 3 \mathrm{H}, \mathrm{CH}$,

$\operatorname{Ar}-H), 6.13(\mathrm{~d}, 1 \mathrm{H}, J=16.0 \mathrm{~Hz}, \mathrm{CH}), 5.94$ (brs, $1 \mathrm{H}, \mathrm{NH}), 4.35\left(\mathrm{~d}, 2 \mathrm{H}, J=5.5 \mathrm{~Hz}, \mathrm{CH}_{2}\right.$ ), $3.80\left(\mathrm{~s}, 3 \mathrm{H}, \mathrm{OCH}_{3}\right), 2.95\left(\mathrm{t}, 2 \mathrm{H}, J=7.0 \mathrm{~Hz}, \mathrm{CH}_{2}\right), 2.51\left(\mathrm{t}, 2 \mathrm{H}, J=6.5 \mathrm{~Hz}, \mathrm{CH}_{2}\right), 2.36(\mathrm{t}$, $\left.2 \mathrm{H}, J=7.0 \mathrm{~Hz}, \mathrm{CH}_{2}\right), 2.25\left(\mathrm{q}, 2 \mathrm{H}, J=7.0 \mathrm{~Hz}, \mathrm{CH}_{2}\right), 1.47-1.53\left(\mathrm{~m}, 2 \mathrm{H}, \mathrm{CH}_{2}\right), 1.61(\mathrm{~m}, 2 \mathrm{H}$, $\left.\mathrm{CH}_{2}\right)$, 1.65-1.71 (m, 2H, $\left.\mathrm{CH}_{2}\right) ;{ }^{13} \mathrm{C} \mathrm{NMR}\left(100 \mathrm{MHz} \mathrm{CDCl}_{3}\right) \delta$ 199.0, 171.8, 158.9, 147.0, 130.4, 130.3, 129.0 (2C), 119.5, 114.0 (2C), 55.3, 43.1, 35.1, 32.0, 30.0, 28.1, 27.2, 25.1, 17.0; MS (ESI) Exact mass calculated for $\mathrm{C}_{20} \mathrm{H}_{26} \mathrm{~N}_{2} \mathrm{O}_{3}(\mathrm{M}+\mathrm{H})^{+}:$343.1943. Found: 343.1904 .
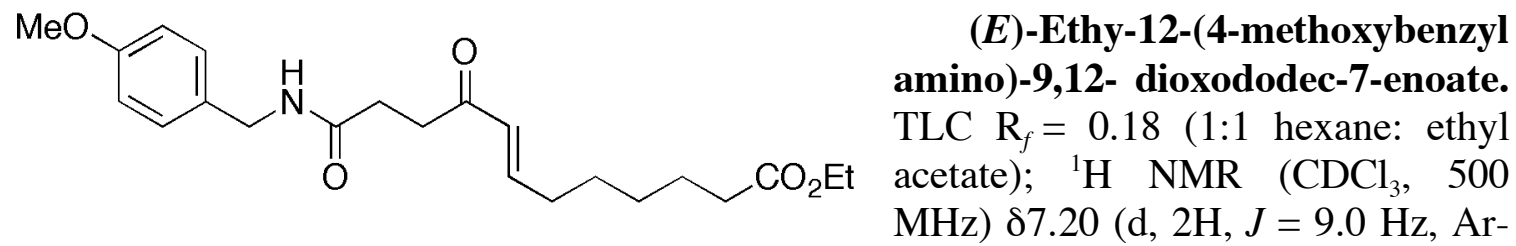

$H)$, 6.84-6.90 (m, 3H, CH, Ar-H), 6.13 (d, $1 \mathrm{H}, J=16.0 \mathrm{~Hz}, \mathrm{CH}), 5.99$ (brs, $1 \mathrm{H}, \mathrm{N} H$ ), $4.35\left(\mathrm{~d}, 2 \mathrm{H}, J=5.5 \mathrm{~Hz}, \mathrm{CH}_{2}\right), 4.10-4.15\left(\mathrm{~m}, 2 \mathrm{H}, \mathrm{CH}_{2}\right), 3.80\left(\mathrm{~s}, 3 \mathrm{H}, \mathrm{OCH}_{3}\right), 2.95(\mathrm{t}, 2 \mathrm{H}, J$ $\left.=6.5 \mathrm{~Hz}, \mathrm{CH}_{2}\right), 2.44-2.54\left(\mathrm{~m}, 4 \mathrm{H}, \mathrm{CH}_{2}\right), 2.28-2.35\left(\mathrm{~m}, 2 \mathrm{H}, \mathrm{CH}_{2}\right), 1.60-1.67\left(\mathrm{~m}, 2 \mathrm{H}, \mathrm{CH}_{2}\right)$, 1.42-1.54 (m, 2H, $\left.\mathrm{CH}_{2}\right), 1.32-1.40\left(\mathrm{~m}, 2 \mathrm{H}, \mathrm{CH}_{2}\right), 1.23-1.28\left(\mathrm{~m}, 3 \mathrm{H}, \mathrm{CH}_{3}\right) ;{ }^{13} \mathrm{C} \mathrm{NMR}(100$ $\left.\mathrm{MHz}, \mathrm{CDCl}_{3}\right) \delta$ 199.1, 173.6, 171.8 158.9, 147.8, 130.1, 129.0 (2C), 119.5, 114.0 (2C), 77.0, 60.2, 55.2, 43.0, 35.1, 34.1, 32.2, 30.0, 28.6, 27.6, 24.6, 14.2; MS (ESI) Exact mass calculated for $\mathrm{C}_{22} \mathrm{H}_{31} \mathrm{NO}_{5}(\mathrm{M}+\mathrm{H})^{+}: 390.2202$. Found: 390.2221 .

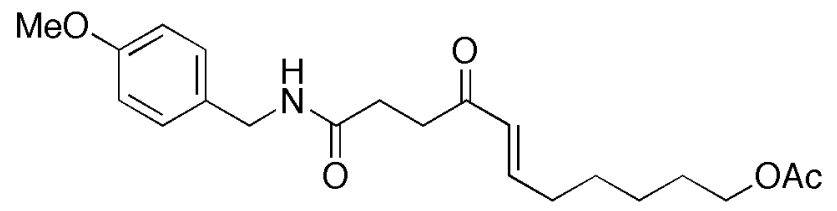

(E)-Ethy-11-(4-methoxybenzyl amino)-8,11-dioxododec-6-enyl acetate. $\mathrm{TLC} \mathrm{R}_{f}=0.13(1: 1$ hexane: ethyl acetate); ${ }^{1} \mathrm{H}$ NMR $\left(\mathrm{CDCl}_{3}, 500\right.$ $\mathrm{MHz}) \delta 7.19(\mathrm{~d}, 2 \mathrm{H}, J=9.0 \mathrm{~Hz}, \mathrm{Ar}-H)$, 6.85-6.89 (m, 3H, CH, Ar-H), 6.13 (d, 1H, J = 16.0 Hz, CH), 6.02 (brs, 1H, NH), 4.35 (d, $\left.2 \mathrm{H}, J=5.5 \mathrm{~Hz}, \mathrm{CH}_{2}\right), 4.06\left(\mathrm{t}, 2 \mathrm{H}, J=6.5 \mathrm{~Hz}, \mathrm{CH}_{2}\right), 3.79\left(\mathrm{~s}, 3 \mathrm{H}, \mathrm{OCH}_{3}\right), 2.95(\mathrm{t}, 2 \mathrm{H}, J=$ $\left.7.0 \mathrm{~Hz}, \mathrm{CH}_{2}\right), 2.50\left(\mathrm{t}, 2 \mathrm{H}, J=7.0 \mathrm{~Hz}, \mathrm{CH}_{2}\right), 2.23\left(\mathrm{q}, 2 \mathrm{H}, \mathrm{J}=7.5 \mathrm{~Hz}, \mathrm{CH}_{2}\right), 2.05(\mathrm{~s}, 3 \mathrm{H}$, $\left.\mathrm{CH}_{3}\right), 1.61-1.67\left(\mathrm{~m}, 2 \mathrm{H}, \mathrm{CH}_{2}\right), 1.47-1.53\left(\mathrm{~m}, 2 \mathrm{H}, \mathrm{CH}_{2}\right), 1.37-1.42\left(\mathrm{~m}, 2 \mathrm{H}, \mathrm{CH}_{2}\right) ;{ }^{13} \mathrm{C}$ NMR (100 MHz, $\left.\mathrm{CDCl}_{3}\right) \delta 199.1,171.8,171.1$ 158.9, 147.6, 130.1, 130.3, 129.0 (2C), 113.9 (2C),64.2, 55.2, 43.0, 35.0, 32.2, 29.9, 28.3, 27.6, 25.5, 20.9; MS (ESI) Exact mass calculated for $\mathrm{C}_{21} \mathrm{H}_{29} \mathrm{NO}_{5}(\mathrm{M}+\mathrm{H})^{+}: 376.2046$. Found: 376.2069 .

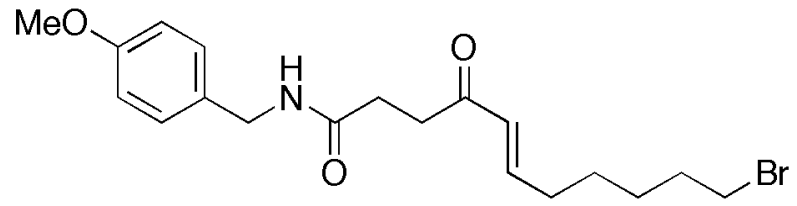

(E)-11-Bromo- $N$-(4-methoxybenzyl)4-oxoundec-5-enamide. TLC $\mathrm{R}_{f}=0.18$ (1:1 hexane: ethyl acetate); ${ }^{1} \mathrm{H}$ NMR $\left(\mathrm{CDCl}_{3}, 500 \mathrm{MHz}\right) \delta 7.19(\mathrm{~d}, 2 \mathrm{H}, J=8.5$ $\mathrm{Hz}, \mathrm{Ar}-\mathrm{H}), 6.84-6.90(\mathrm{~m}, 3 \mathrm{H}, \mathrm{CH}, \mathrm{Ar}-$ $H$ ), 6.12 (d, $1 \mathrm{H}, J=16.5 \mathrm{~Hz}, \mathrm{CH}), 5.95$ (brs, $1 \mathrm{H}, \mathrm{NH}), 4.35$ (d, $2 \mathrm{H}, J=6.0 \mathrm{~Hz}, \mathrm{CH}_{2}$ ), $3.80\left(\mathrm{~s}, 3 \mathrm{H}, \mathrm{OCH}_{3}\right), 3.41\left(\mathrm{t}, 2 \mathrm{H}, J=7.0 \mathrm{~Hz}, \mathrm{CH}_{2}\right), 2.96\left(\mathrm{t}, 2 \mathrm{H}, J=6.5 \mathrm{~Hz}, \mathrm{CH}_{2}\right), 2.51(\mathrm{t}$, 
$\left.2 \mathrm{H}, J=6.5 \mathrm{~Hz}, \mathrm{CH}_{2}\right), 2.24\left(\mathrm{q}, 2 \mathrm{H}, J=6.5 \mathrm{~Hz}, \mathrm{CH}_{2}\right), 1.86-1.89\left(\mathrm{~m}, 2 \mathrm{H}, \mathrm{CH}_{2}\right), 1.48-1.51$ (m, 4H, $\left.\mathrm{CH}_{2}\right) ;{ }^{13} \mathrm{C}$ NMR $\left(100 \mathrm{MHz}, \mathrm{CDCl}_{3}\right) \delta 199.1,171.8$ 158.9, 147.5, 130.3, 130.2, 129.0 (2C), 114.0 (2C), 55.2, 43.1, 35.1, 33.5, 32.4, 32.2, 30.1, 27.7, 27.2; MS (ESI) Exact mass calculated for $\mathrm{C}_{19} \mathrm{H}_{26} \mathrm{BrNO}_{3}(\mathrm{M}+\mathrm{H})^{+}:$396.1096. Found: 396.1076 .

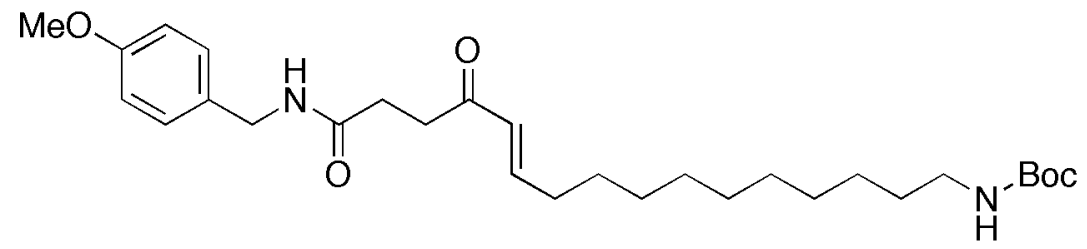

$(\boldsymbol{E})-11-B r o m o-N-(4-$
methoxybenzyl)-4-
oxoundec-5-enamide.
TLC $\mathrm{R}_{f}=0.075(2: 1$
hexane: ethyl acetate);

${ }^{1} \mathrm{H} \mathrm{NMR}\left(\mathrm{CDCl}_{3}, 500 \mathrm{MHz}\right) \delta 7.19$ (d, 2H, $\left.J=9.0 \mathrm{~Hz}, \mathrm{Ar}-H\right), 6.84-6.92(\mathrm{~m}, 3 \mathrm{H}, \mathrm{CH}, \mathrm{Ar}-$ $H$ ), $6.10(\mathrm{~d}, 1 \mathrm{H}, J=15.0 \mathrm{~Hz}, \mathrm{CH}), 5.97$ (brs, $1 \mathrm{H}, \mathrm{NH}), 4.51$ (brs, $1 \mathrm{H}, \mathrm{NH}), 4.35$ (d, $2 \mathrm{H}, J$ $\left.=6.0 \mathrm{~Hz}, \mathrm{CH}_{2}\right), 3.80\left(\mathrm{~s}, 3 \mathrm{H}, \mathrm{OCH}_{3}\right), 3.10\left(\mathrm{br}, 2 \mathrm{H}, \mathrm{CH}_{2}\right), 2.96\left(\mathrm{t}, 2 \mathrm{H}, J=6.5 \mathrm{~Hz}, \mathrm{CH}_{2}\right)$, $2.51\left(\mathrm{t}, 2 \mathrm{H}, J=6.5 \mathrm{~Hz}, \mathrm{CH}_{2}\right), 2.21\left(\mathrm{q}, 2 \mathrm{H}, J=6.5 \mathrm{~Hz}, \mathrm{CH}_{2}\right), 1.60-1.62\left(\mathrm{br}, 2 \mathrm{H}, \mathrm{CH}_{2}\right), 1.44$ (brs, 9H, $\left.\mathrm{C}\left(\mathrm{CH}_{3}\right)_{3}\right), 1.28$ (brs, $\left.14 \mathrm{H}, \mathrm{CH}_{2}\right) ;{ }^{13} \mathrm{C} \mathrm{NMR}\left(100 \mathrm{MHz}, \mathrm{CDCl}_{3}\right) \delta 199.2,171.9$ 158.9, 148.3, 130.4, 130.0, 129.0 (2C), 114.0 (2C), 78.1, 55.2, 43.1, 40.6, 35.0, 32.5, 30.1, 30.0, 29.4, 29.3 (2C), 29.2 (2C), 29.1, 28.4 (3C), 28.0, 26.8; MS (ESI) Exact mass calculated for $\mathrm{C}_{29} \mathrm{H}_{46} \mathrm{NO}_{5}(\mathrm{M}+\mathrm{H})^{+}:$503.3407. Found: 503.2653.

\section{${ }^{17}$ O NMR spectrum}

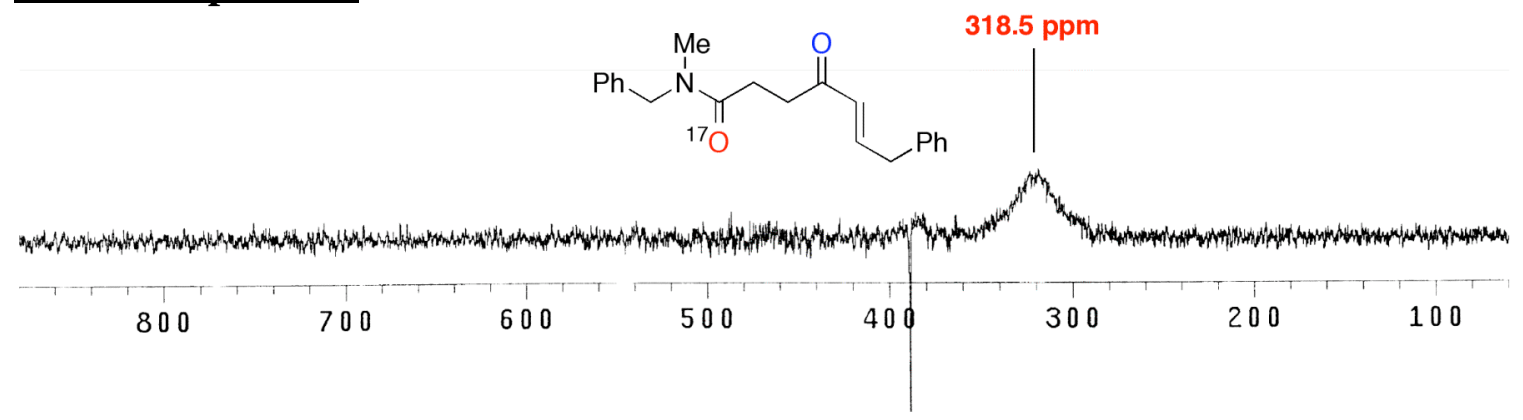

${ }^{1} \mathrm{H}$ and ${ }^{13} \mathrm{C}$ NMR spectrum for the above compounds appear on the following pages. 
$\mathrm{MeO}$

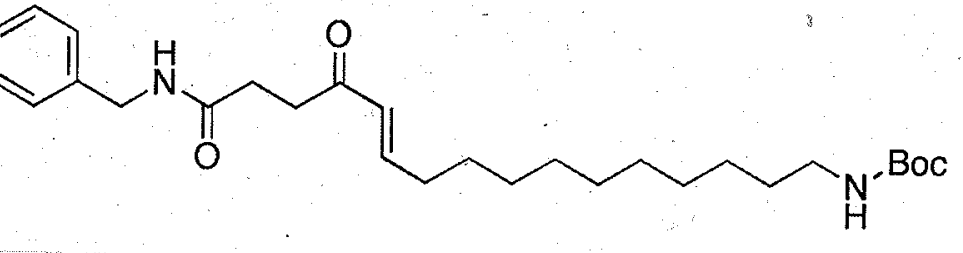

Pulse 79.4 degrees

Acq. time 2.048
width $8000.0 \mathrm{~Hz}$

16 repetitions

OBSERVE H1, 499.6156699 MHZ

DATA PROCESSING

line broadening $0.1 \mathrm{~Hz}$

Total time 0 min, $40 \mathrm{sec}$

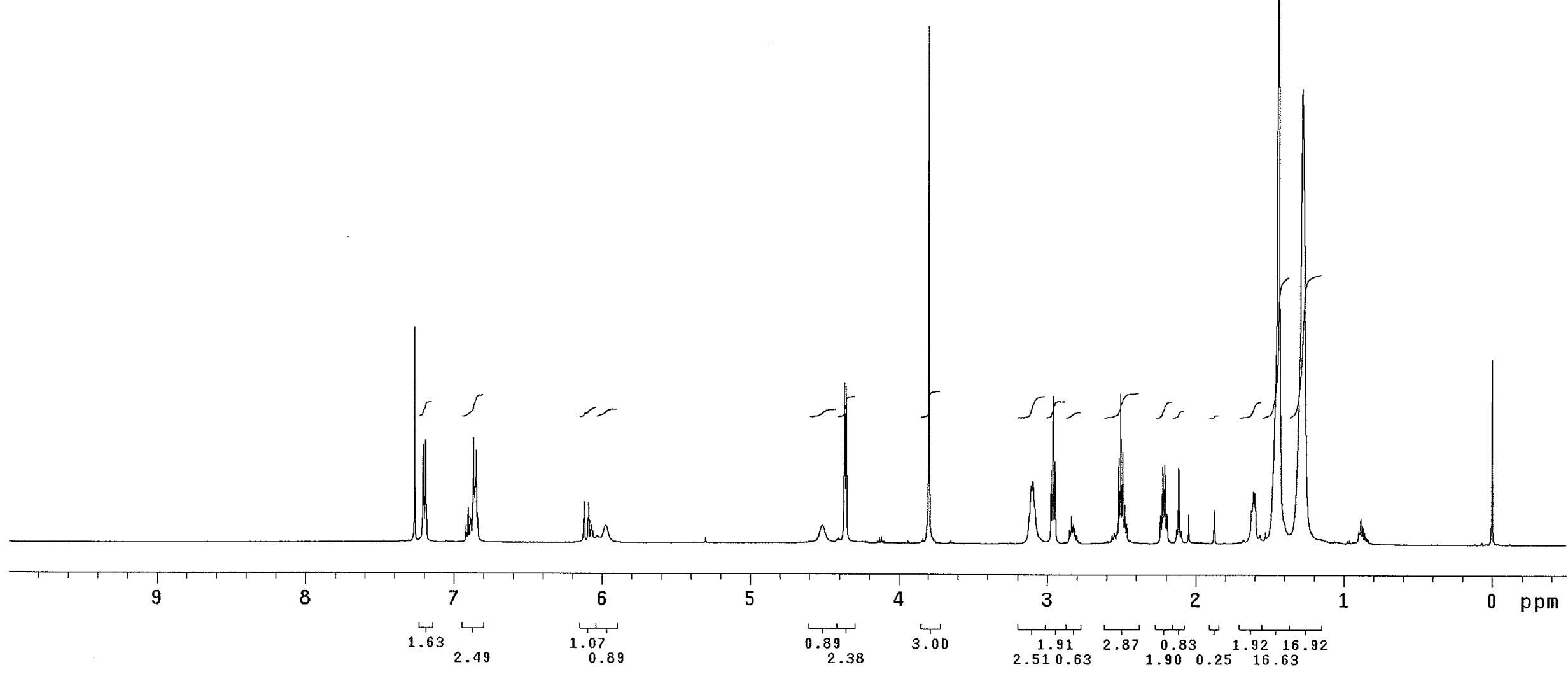




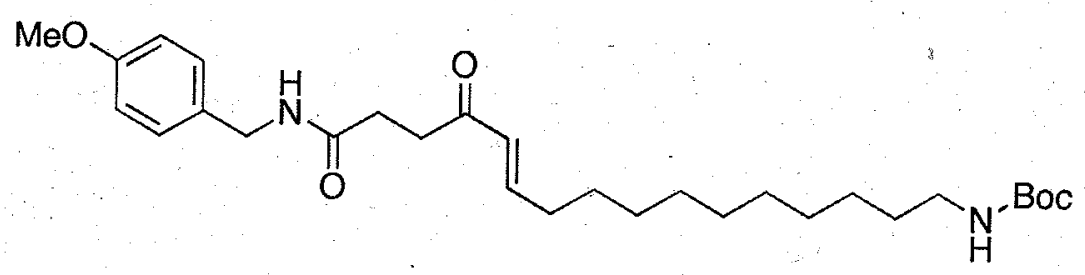

Pulse 40.0 degrees

Width $29996.3 \mathrm{~Hz}$

10000 repetition

OBSERVE C13, $125.6284643 \mathrm{MHZ}$

DECOUPLE H1, 499.6181772 MHZ

cont inuous ly on

WALT2-16 modulate

Line broadening $1.0 \mathrm{~Hz}$

Total time $3 \mathrm{hr}, 3 \mathrm{~min}, 33 \mathrm{sec}$

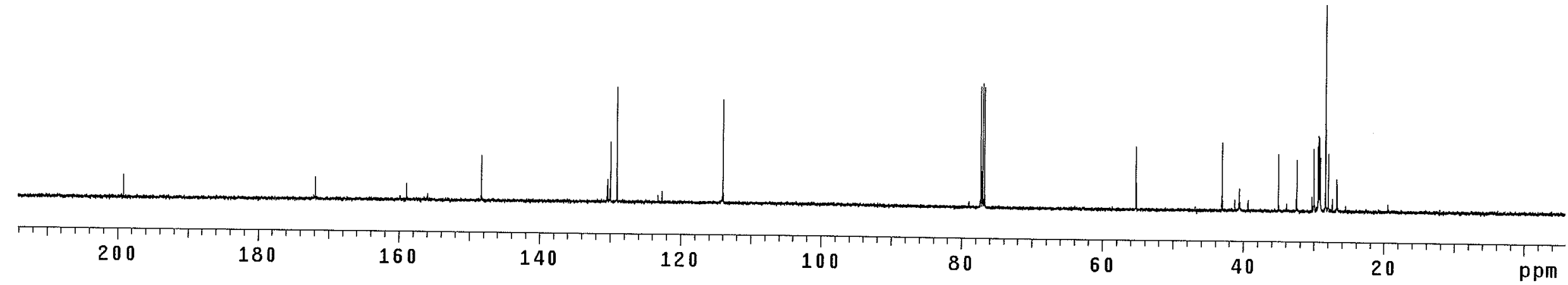




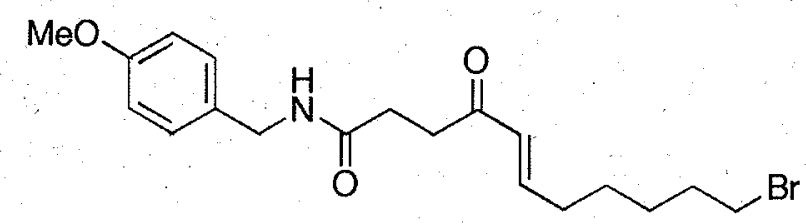

Relax. de lay $0.200 \mathrm{sec}$

Acq time $2.048 \mathrm{sec}$

Acq time 2.048 sec
width $8000.0 \mathrm{~Hz}$

16 repetitions

OBSERVE H1, 499.6156699 MHZ

Line broadening $0.1 \mathrm{~Hz}$

FT size 32768
Total time $0 \mathrm{~min}, 40 \mathrm{sec}$

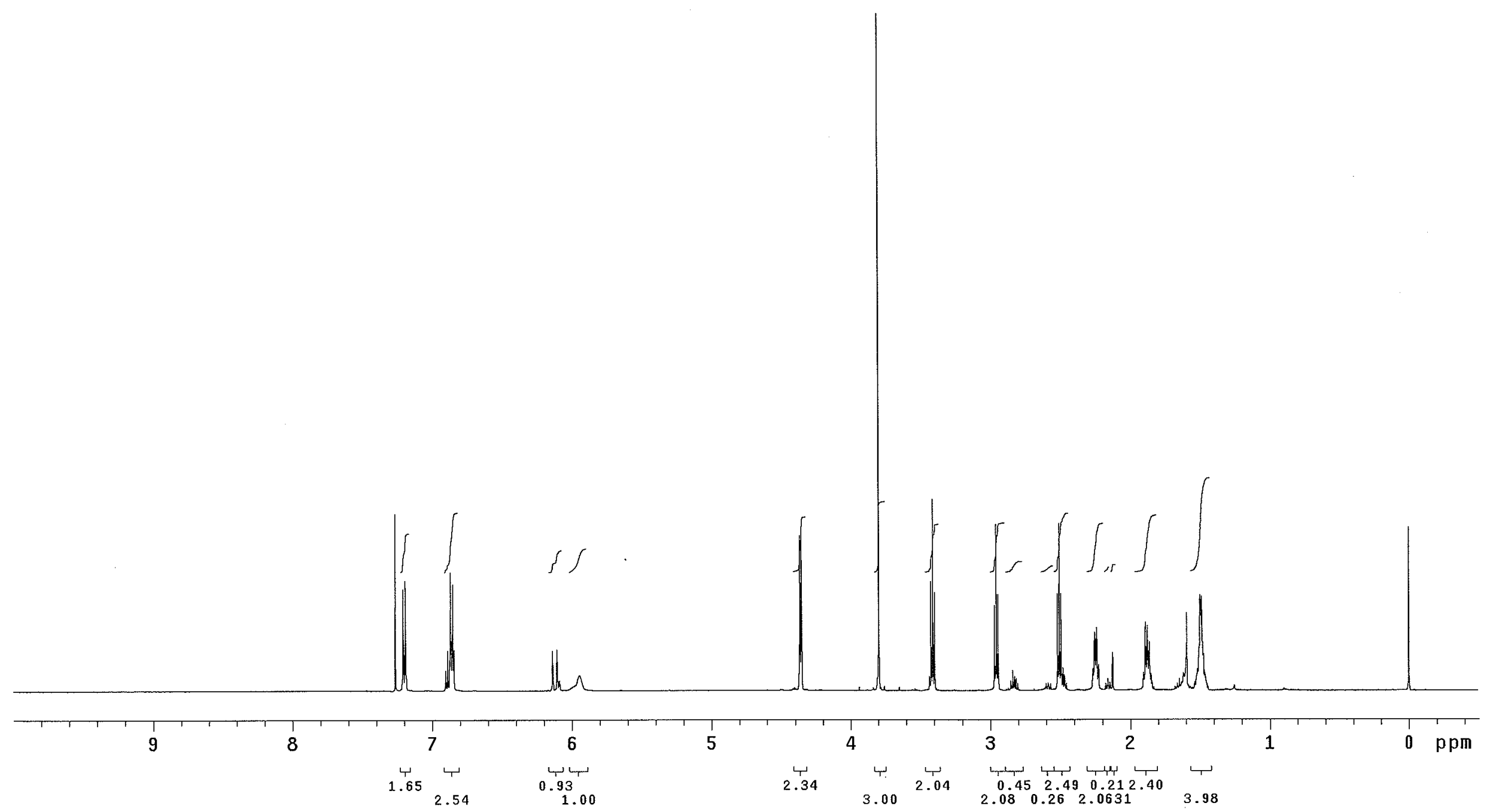




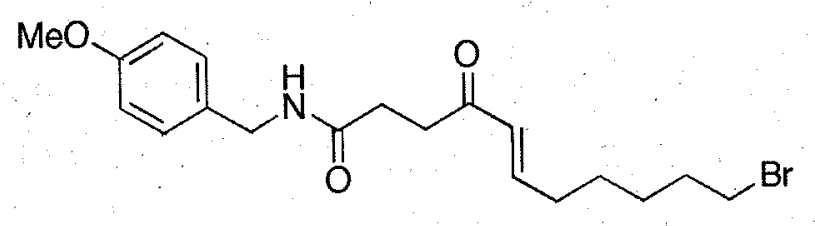

Fi1e: $6904-4-13 \mathrm{C}$

Pulse sequence: s2put

Solvent: $\mathrm{CDC} 13$

Temp. $23.0 \mathrm{C} / 296.1 \mathrm{~K}$

Pulse 40.0 degrees

Acq. time $1.092 \mathrm{sec}$

Width $29996.3 \mathrm{~Hz}$

100000 repetitions

OBSERVE C13, $125.6284634 \mathrm{MHz}$

POWer 34 A

cont inuous Ty on

WALT2-16 modulated

Line broadening 1.0

Total time $30 \mathrm{hr}, 35 \mathrm{~min}$

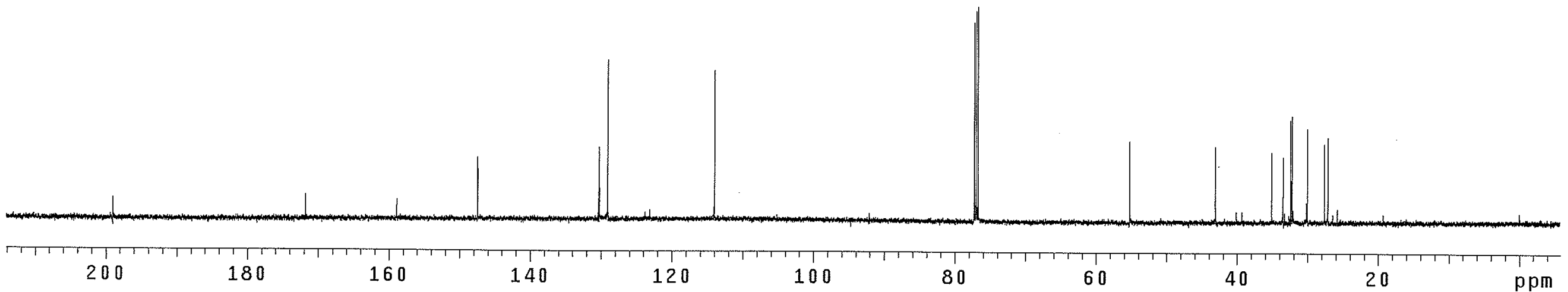




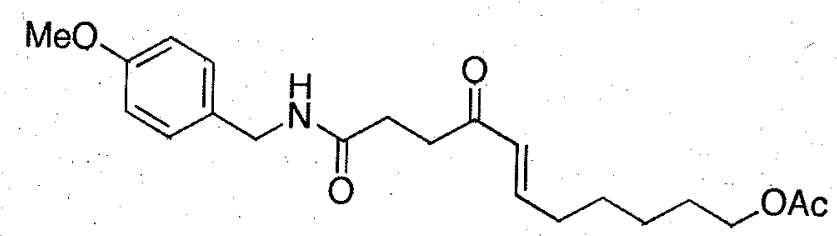

Relax. de lay u.zuu jou

Acg tim 2.048 sec

width $8000.0 \mathrm{~Hz}$

16 repetitions
OBSERVE H1, 499.6156674 MHz

Line broadening $0.1 \mathrm{~Hz}$

Total time $0 \mathrm{~min}, 40 \mathrm{sec}$

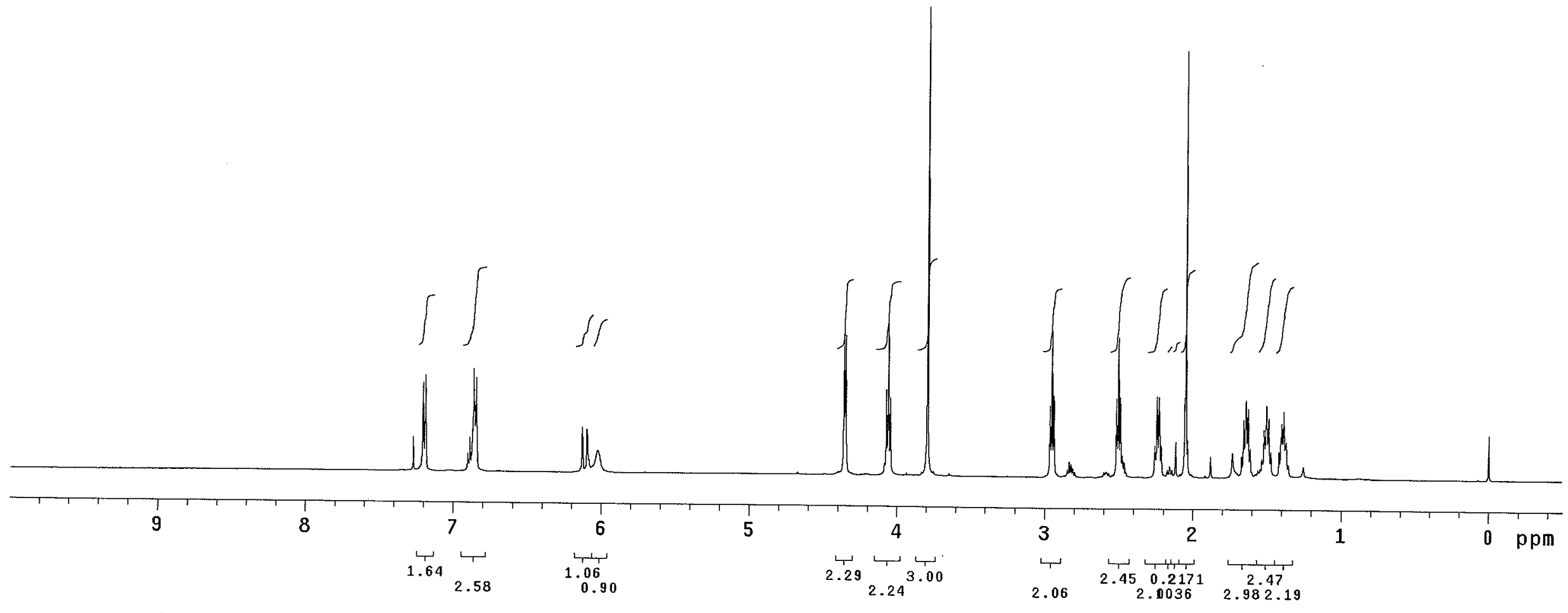




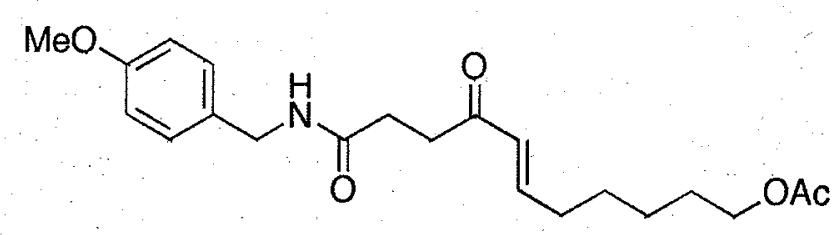

Pulse 40.0 degrees

Acq. time $1.092 \mathrm{sec}$

1000 repetitions

OBSERVE C13, 125.6284689 MHZ

DECOUPLE HI, 499.6181772 MHz

continuous Ty

WALTZ-16 modulated

Line broadening $1.0 \mathrm{~Hz}$

Total time $3 \mathrm{hr}, 3 \mathrm{~min}, 33 \mathrm{sec}$

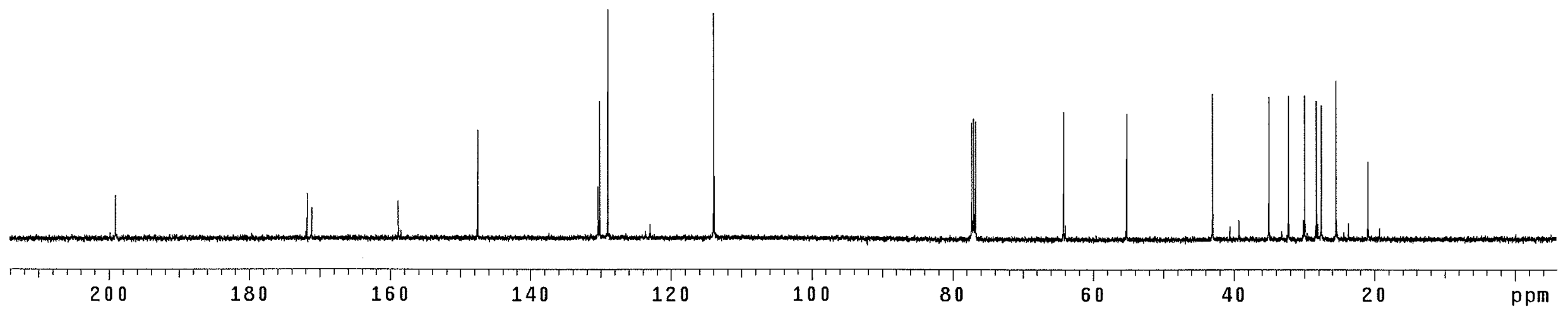




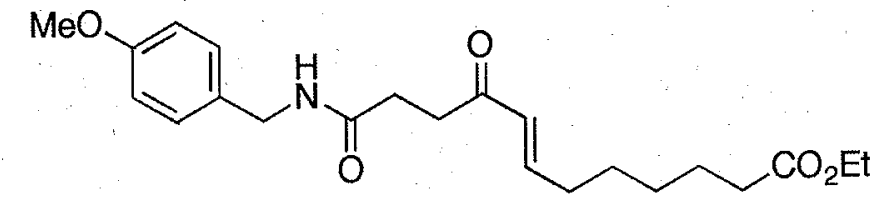

Relax. delay $0.200 \mathrm{sec}$

Acq time $2.048 \mathrm{sec}$

Width $8000.0 \mathrm{~Hz}$

16 repetitions

OBSERVE H1, 499.6156684 MHZ

Line broadening $0.1 \mathrm{~Hz}$

FT size 32768
Total time $0 \mathrm{~min}, 40 \mathrm{sec}$

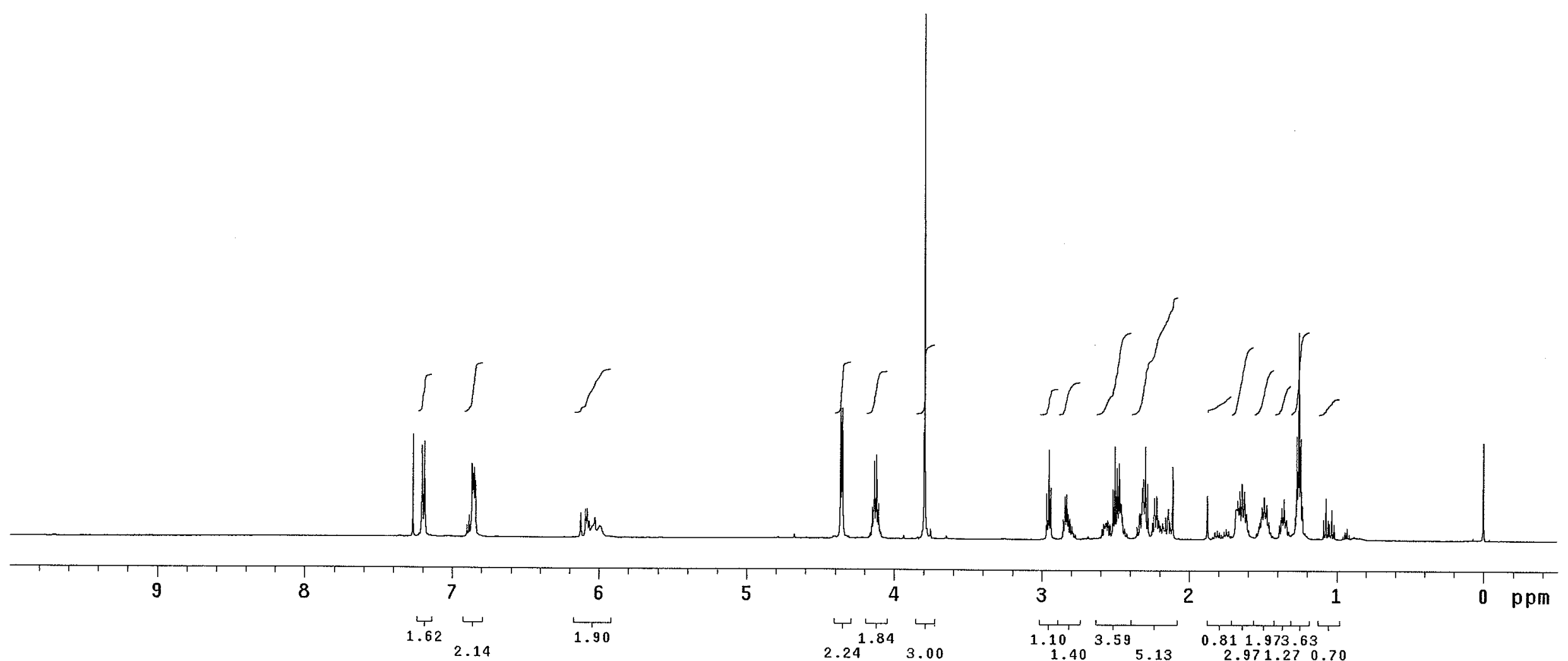




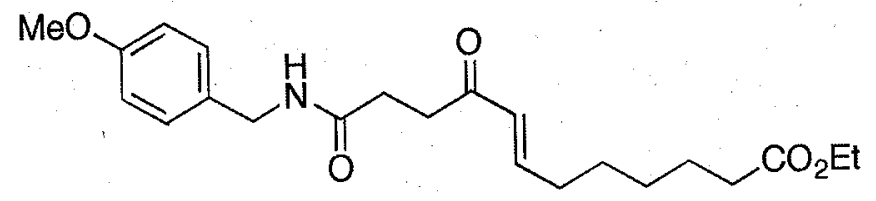

Pulse 40.0 degrees

Acq. time $1.092 \mathrm{sec}$

6892 repetitions

OBSERVE C13, 125.6284653 MHZ

DECOUPLE H1, 499.6181772 MHZ

Power $34 \mathrm{~dB}$

WALTZ-16

DATA PROCESSING

Line broadening $1.0 \mathrm{~Hz}$

Total time $3 \mathrm{hr}, 3 \mathrm{~min}, 33 \mathrm{sec}$

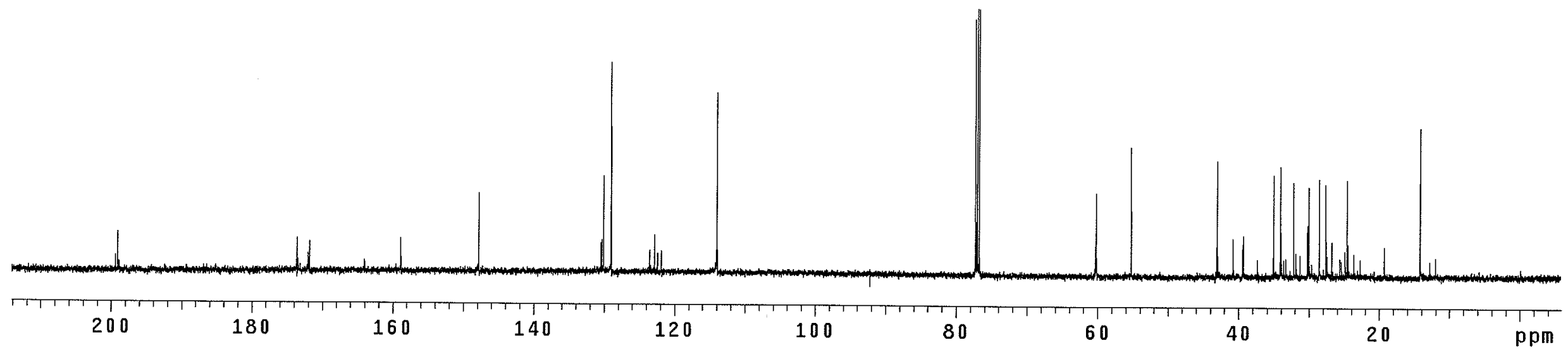




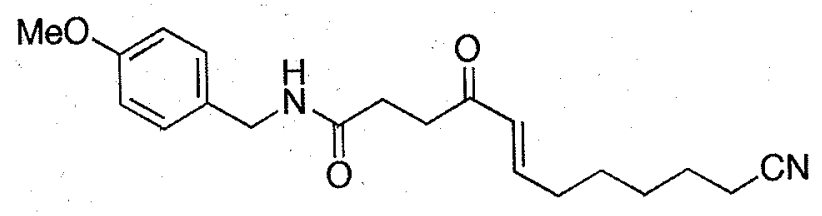

Relax. de lay $0.200 \mathrm{sec}$

Pulse 79.4 degrees

Acq. time 2.048 sec

16 repetitions

OBSERVE H1, 499.6156704 MHz

DATA PROCESSING

Line broadening $0.1 \mathrm{~Hz}$

Total time $0 \mathrm{~min}, 40 \mathrm{sec}$

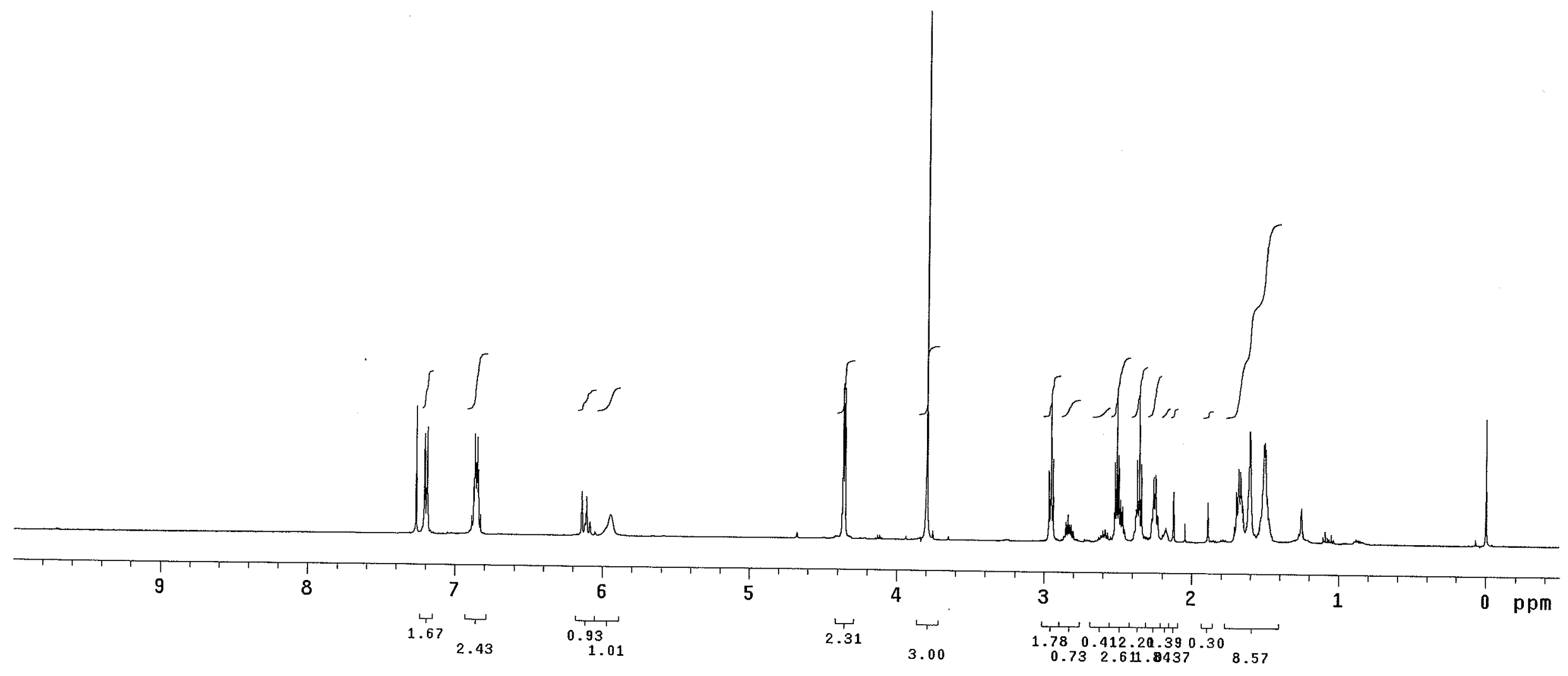




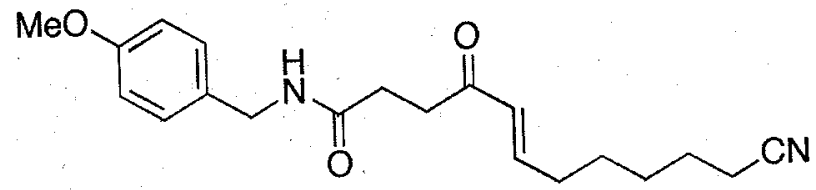

Pulse 40.0 degrees

Acq in time $19996.09 \mathrm{~Hz}$

25523 repetitions

OBSERVE C13, $125.6284653 \mathrm{MHZ}$

Power $34 \mathrm{~dB}$

cont inuous ly on

Line broadening $1.0 \mathrm{~Hz}$

T size 65536

Total time $30 \mathrm{hr}, 35 \mathrm{~min}, 30 \mathrm{sec}$

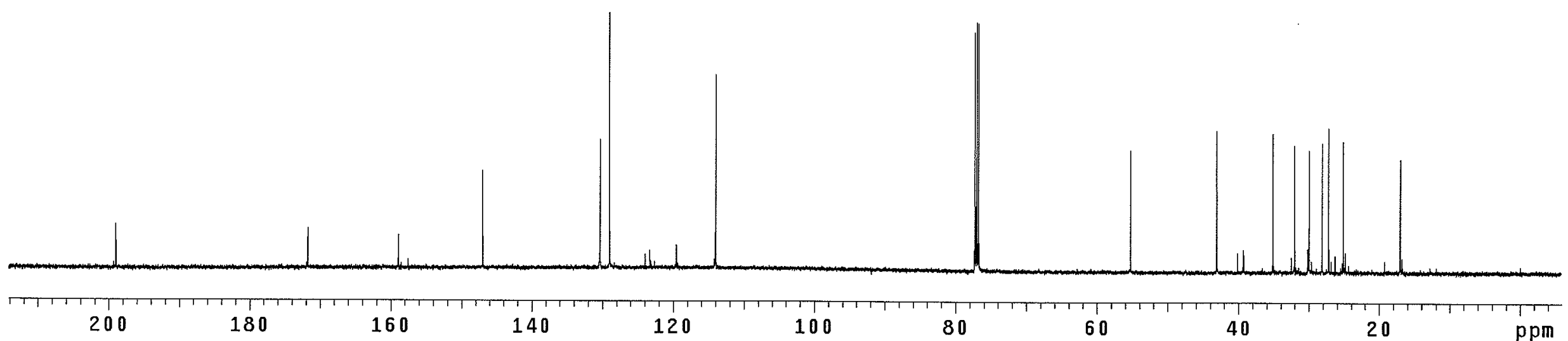




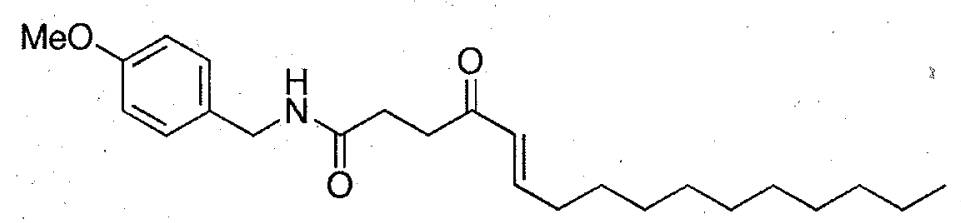

Relax. delay $0.200 \mathrm{sec}$

Pulse 79.4 degrees

Acq time $2.048 \mathrm{sec}$
width $8000.0 \mathrm{~Hz}$

16 repetitions

OBSERVE H1, 499.6156694 MHZ

DATA PROCESSING

Line broadening $0.1 \mathrm{~Hz}$

Total time 0 min, $40 \mathrm{sec}$

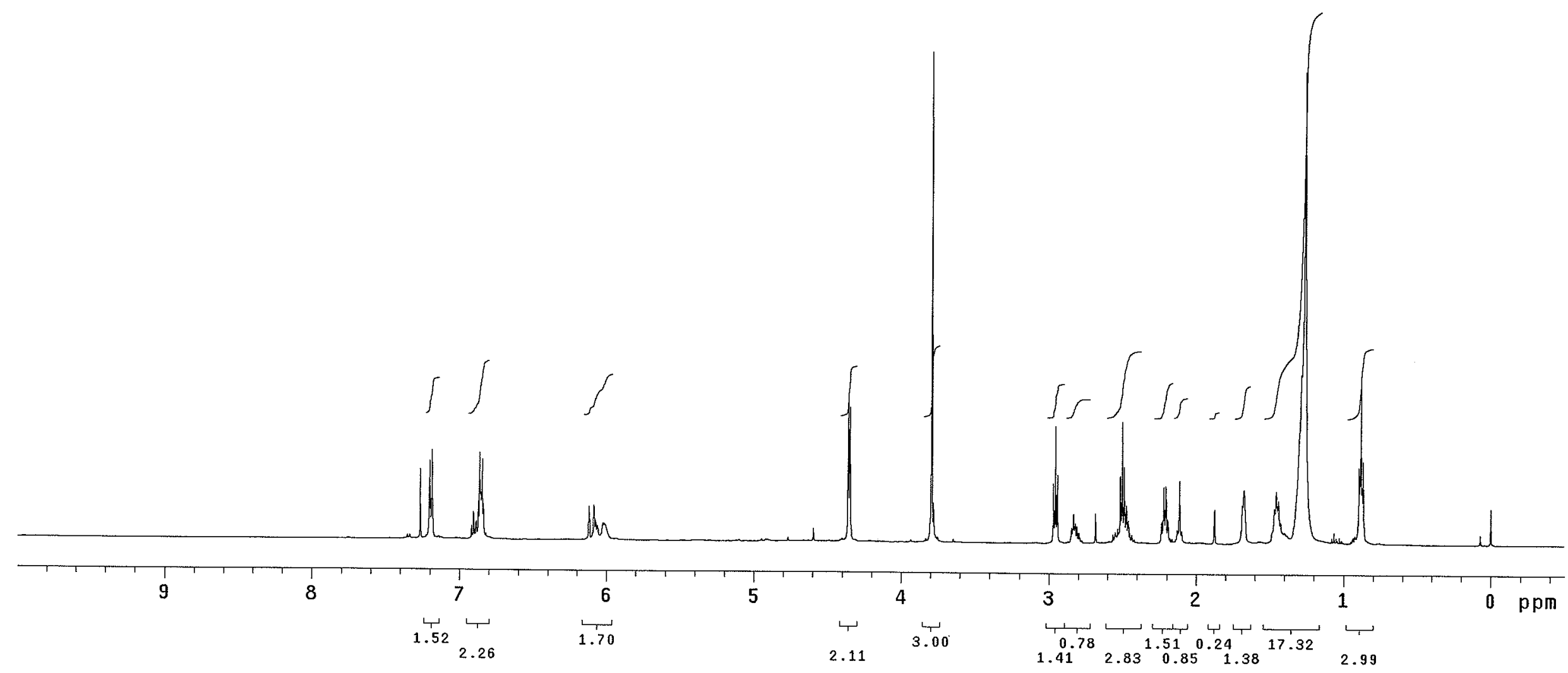




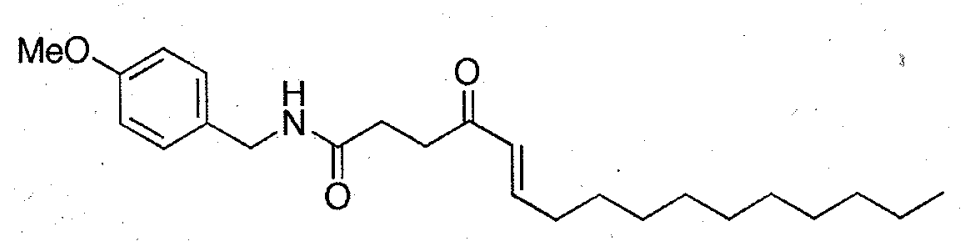

\section{Pulse 40.0 degrees
Acq. time $1.092 \mathrm{sec}$ \\ Acq. time $1.092 \mathrm{sec}$}

25720 repetitions

OBSERVE C13, $125.6284625 \mathrm{MHz}$

Power $34 \mathrm{~dB}$

Cont inuousiy on
WALTZ-16 modulated

Line broadening $1.0 \mathrm{~Hz}$

FT size 65536
Total time $30 \mathrm{hr}, 35 \mathrm{~min}, 30 \mathrm{sec}$

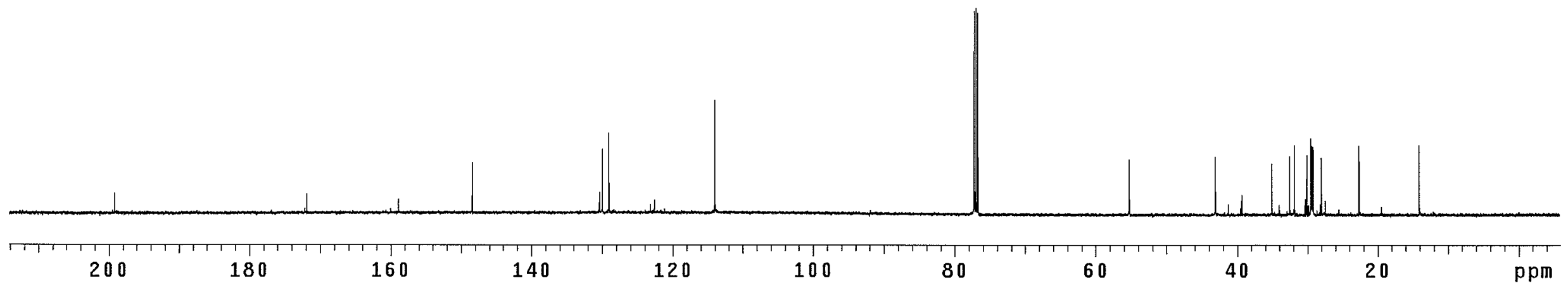




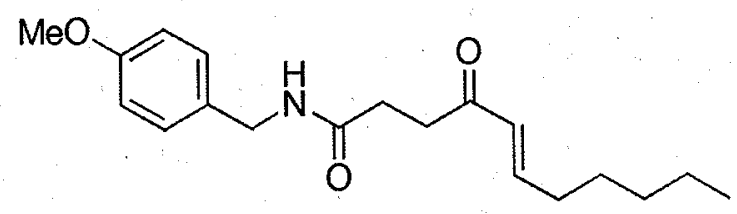

Puise 79.4 degrees

Acq. time $2.048 \mathrm{sec}$

Width $8000.0 \mathrm{~Hz}$
16 repetitions

16 repetitions
OBSERVE H1, 499.6156699 MHz

DATA PROCESSING

Line broadening $0.1 \mathrm{~Hz}$

FT size 32768
Total time $0 \mathrm{~min}, 40 \mathrm{sec}$

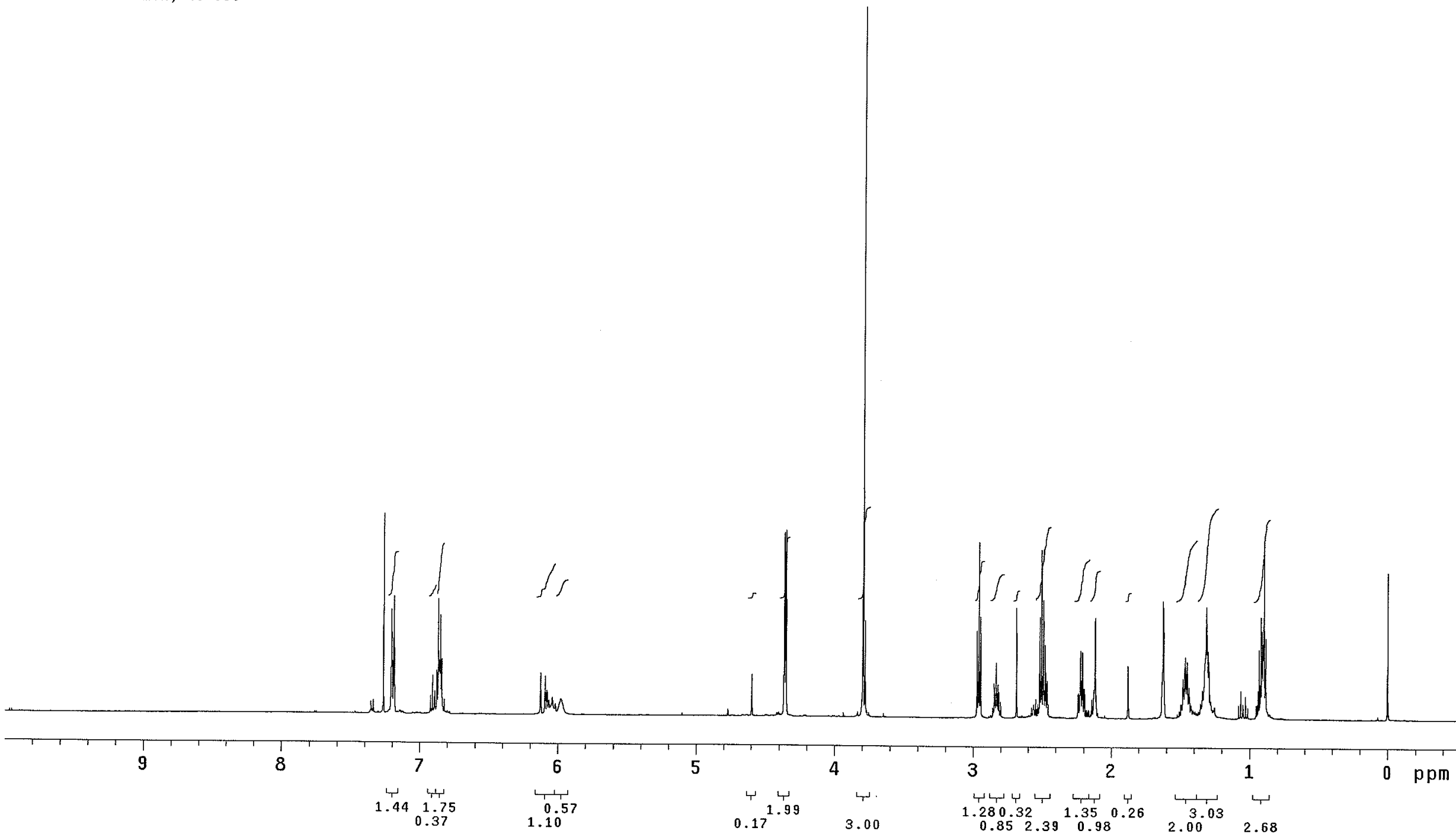




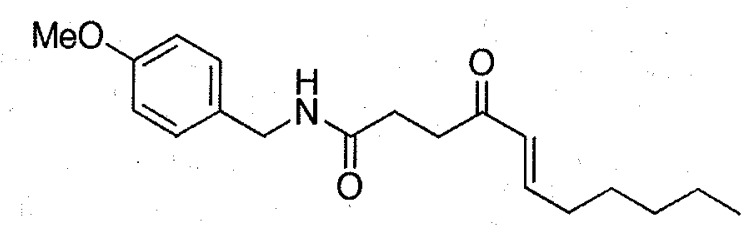

Pulse 40.0 degrees
Acq. time 1.092 sec

Acq. time $1.092 \mathrm{sec}$

23735 repetititz

OBSERVE C13, 125.6284616

DECOUPLE H1, 499.6181772 MHZ

Power $34 \mathrm{~dB}$

WALT $2-16$ modulated

DATA PROCESSING

Line broadening $1.0 \mathrm{~Hz}$

Total time $30 \mathrm{hr}, 35 \mathrm{~min}, 30 \mathrm{sec}$

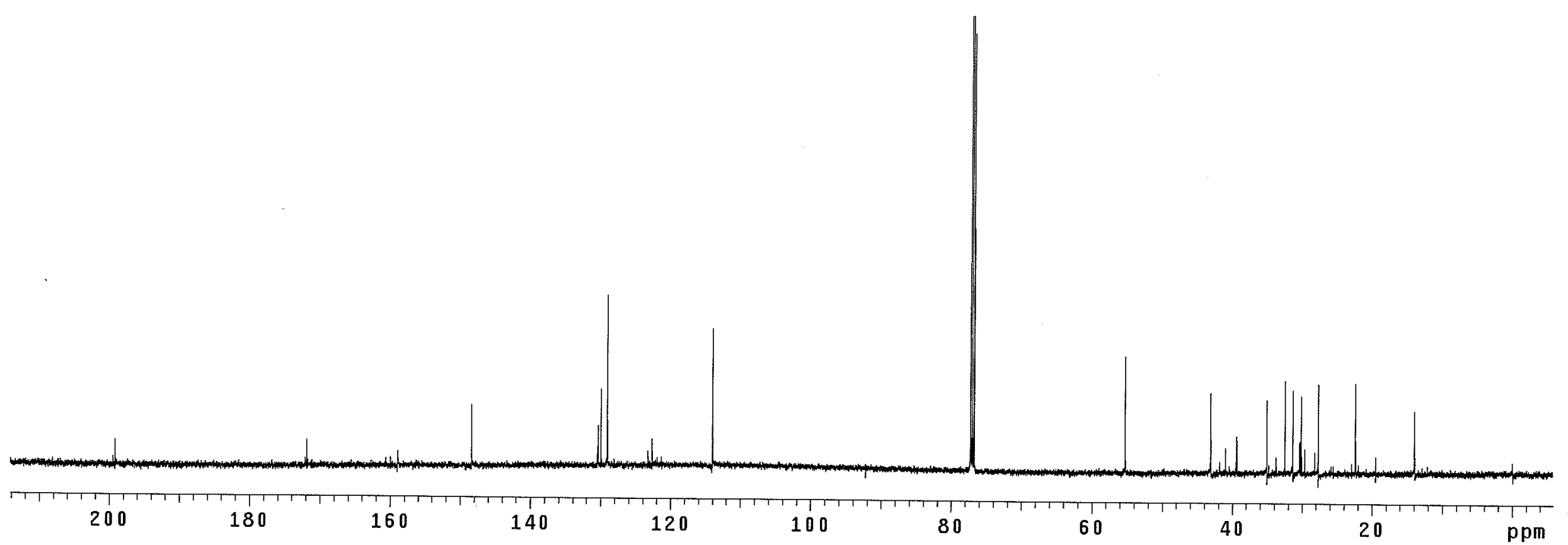


$\mathrm{MeO}$

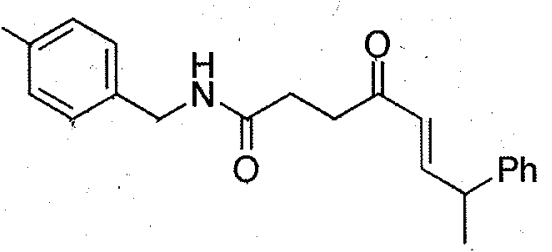

File: 6901-2-1H

Pulse Sequence: s2pur

Solvent: $\mathrm{CDC}_{13}$

Temp. $23.0 \mathrm{C} / 296.1 \mathrm{~K}$

Relax. delay $0.200 \mathrm{sec}$

Pulse 79.4 degrees
Acq. time $2.048 \mathrm{sec}$

width $8000.0 \mathrm{~Hz}$

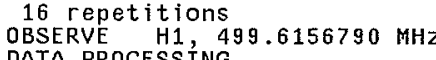

Line broadening $0.1 \mathrm{~Hz}$

FT size 32768

Total time 0 min

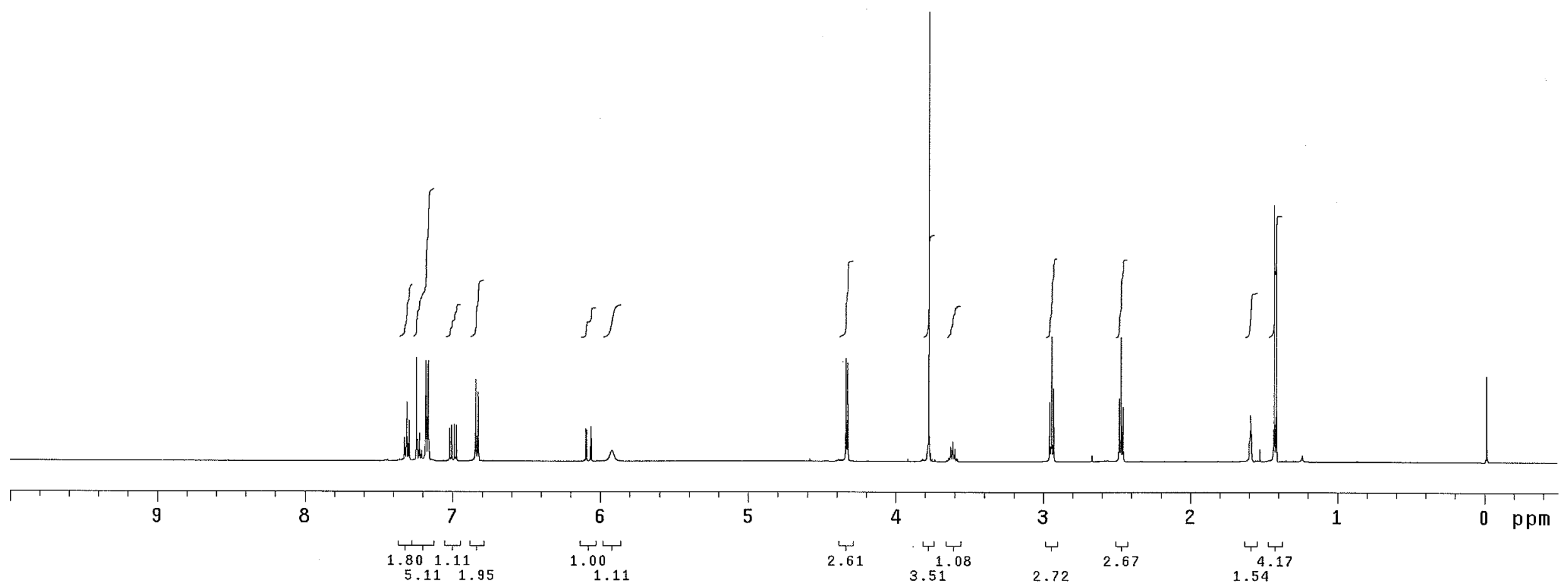




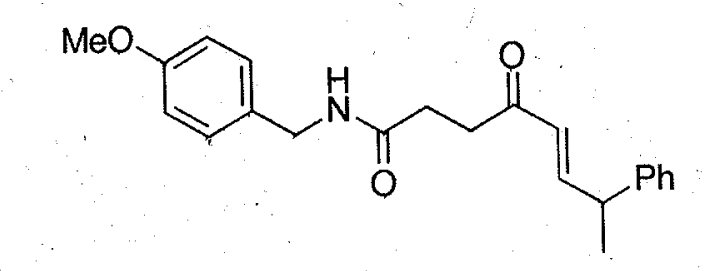

TTе: b901-z-13C

Pulse Sequence: s2pul
Solvent: $C D C 13$

Temp. $23.0 \mathrm{C} / 296.1 \mathrm{~K}$

Pulse 40.0 degrees

Acq time $1.092 \mathrm{sec}$

Width $29996.3 \mathrm{~Hz}$

repetition

OECOUPL C13, $125.6284616 \mathrm{MHZ}$

Power $34 \mathrm{~dB}$

WALTZ-16

WALTZ-16 modu lat
DATA PROGESSING

Line broadening $1.0 \mathrm{~Hz}$

Total time $30 \mathrm{hr}, 35 \mathrm{~min}$

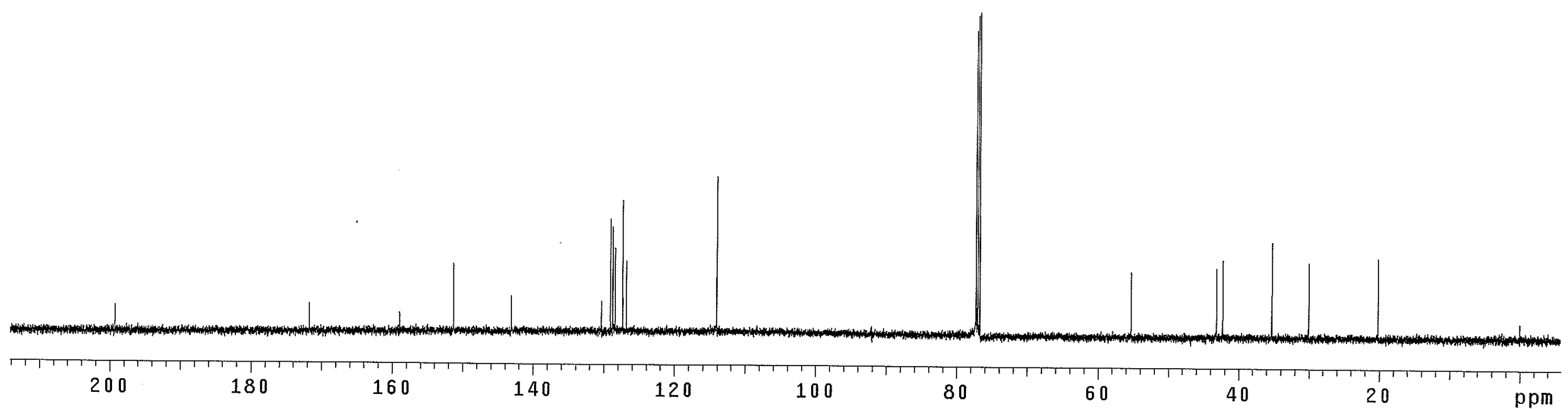




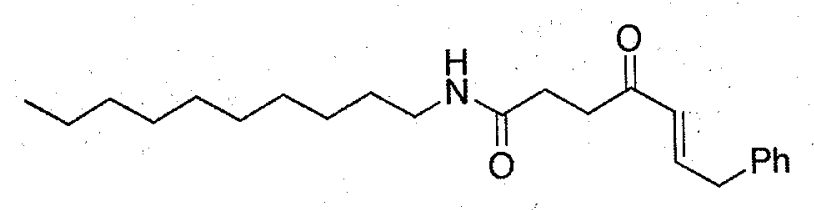

Re lax. de lay $0.200 \mathrm{sec}$

Putse 79.4 degrees

Acq. time 2.048 sec

16 repetitions

OBSERYE H1, 499.6156689 MHZ

DATA PROCESSING

Li size broadening $0.1 \mathrm{~Hz}$

Total time $0 \mathrm{~min}, 40 \mathrm{sec}$

.




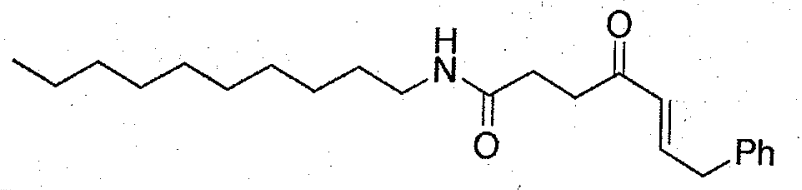

Samp re arrectory:

File: 6901-1-13C

Pulse sequence: s2pur
Solvent: $C D C 13$

Temp. $23.0 \mathrm{C} / 296.1$

Pulse 40.0 degrees

Acq time 1.092 sec

Width $29996.3 \mathrm{~Hz}$

OBSERVE C13, 125.6284653 MHZ

DECOUPLE H1, 499.6181772 MHZ

Power 34 dB

DATA PROCESSING

Line broadening $1.0 \mathrm{~Hz}$

FT size 65536

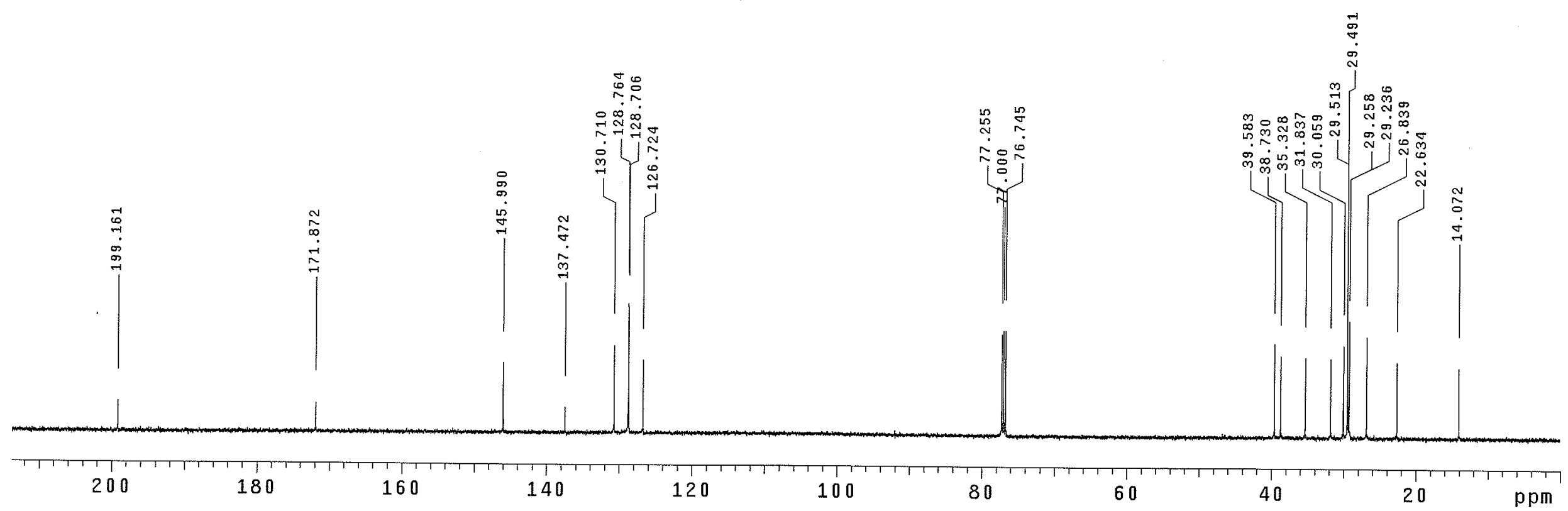




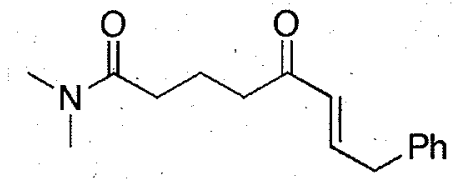

Re lax. de lay 0.200 sec

Pulse 79.4 degrees

Ach time 2.048 sec

16 repetitions

OBSERVE H1, 499.6156790 MHZ

DATA PROCESSING

FT sine broadening $0.1 \mathrm{~Hz}$

Total time 0 min, $40 \mathrm{sec}$

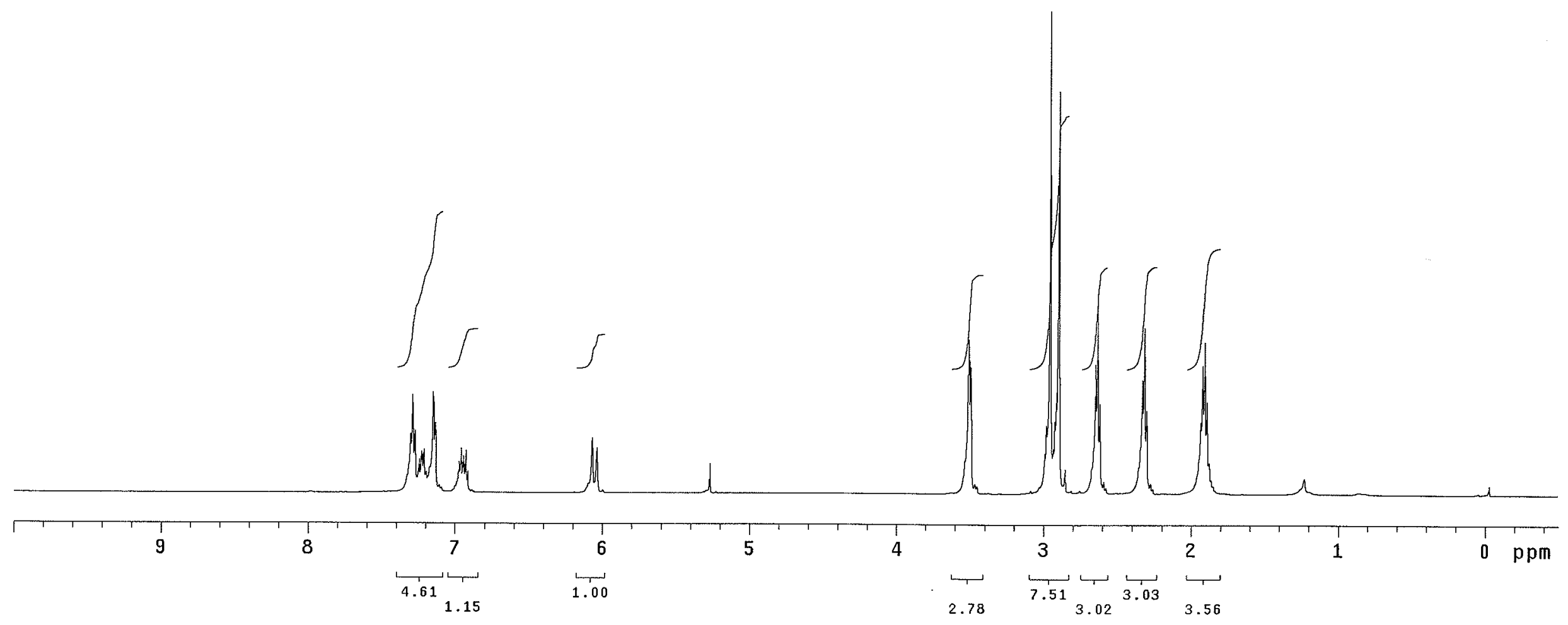




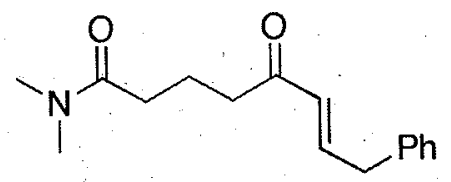

INOVA-500 "inova500"

Pulse 40.0 degrees

Acq. time $1.092 \mathrm{sec}$

$6.3 \mathrm{~Hz}$

OBSERVE C13, 125.6284680 MHz

DECOUPLE H1, 499.6181772 MHZ

cont inuous ly

WALTZ-16 ly on

DATA PROCESSING

Line broadening $1.0 \mathrm{~Hz}$

Total time $30 \mathrm{hr}, 35 \mathrm{~min}, 30 \mathrm{sec}$

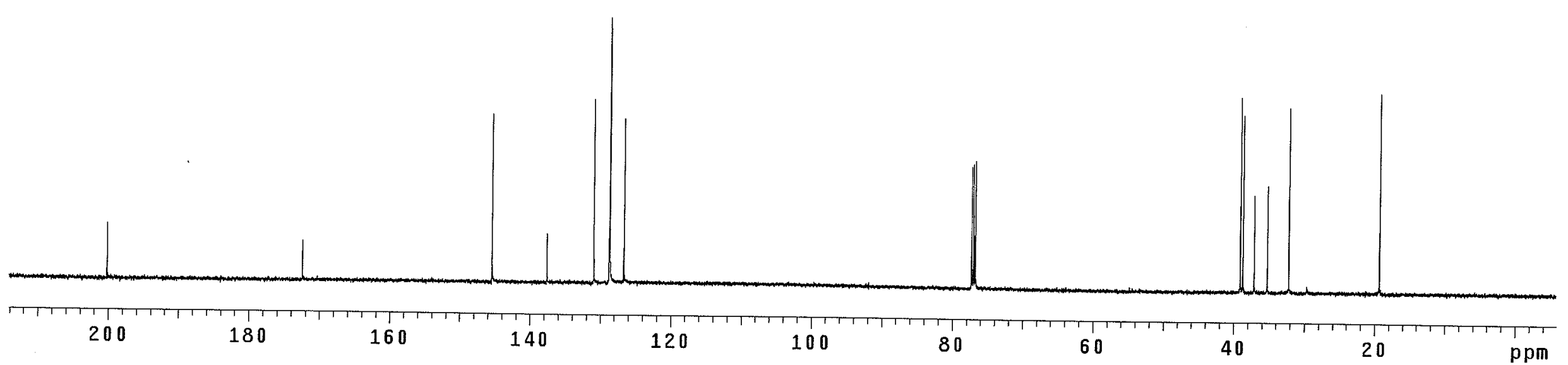




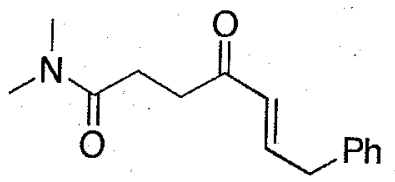

INOVA-500 "i nova 500 "

Re lax. de lay $0.200 \mathrm{sec}$
Pulse 79.4 degrees

Acq. time $2.048 \mathrm{sec}$

Width $8000.0 \mathrm{~Hz}$

OBSERVE H1,499.6156704 MHZ

DATA PROCESSING

ine broadening $0.1 \mathrm{~Hz}$

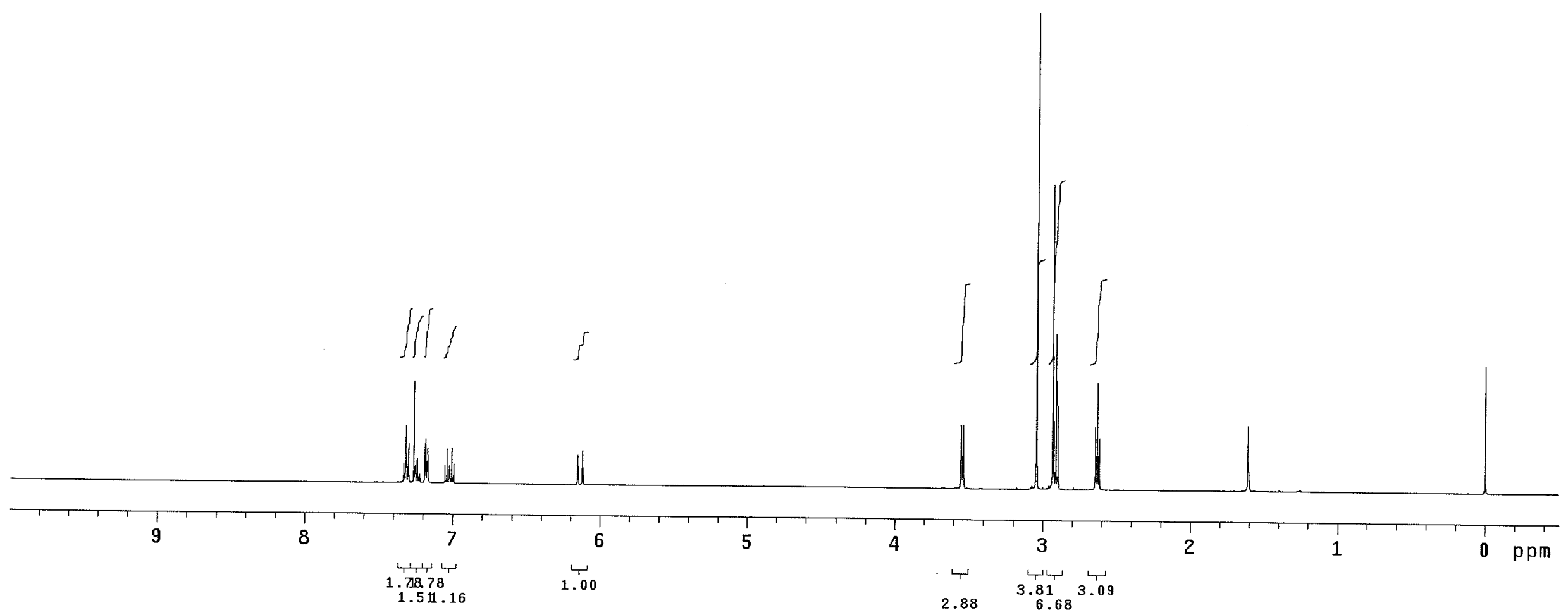




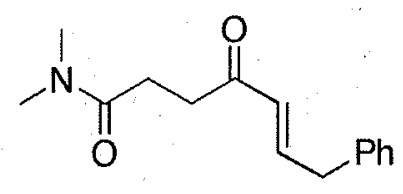

INOVA-500 "I-

Pulse 40.0 degrees

Acq. time $1.092 \mathrm{sec}$

5390 repetition

OBSERVE C13, $125.6284616 \mathrm{MHZ}$

cont inuous Iy

WALTZ-16 modulate

DAT prossing $1.0 \mathrm{~Hz}$

FT size 6553

Total time $30 \mathrm{hr}, 35 \mathrm{~min}, 30 \mathrm{sec}$

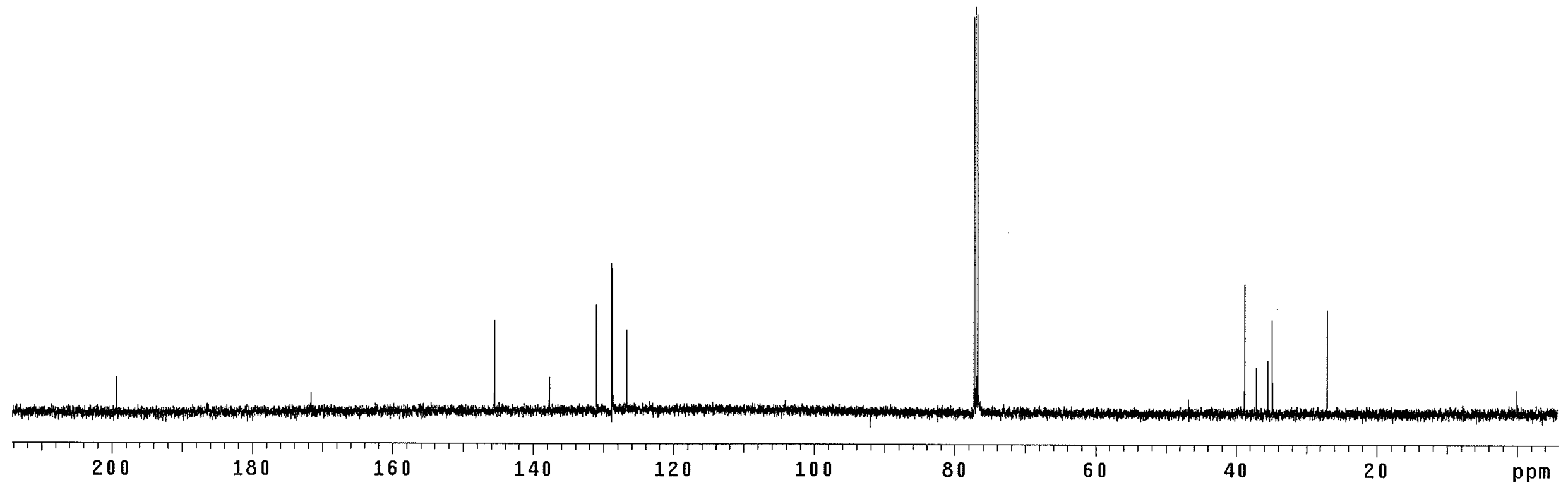




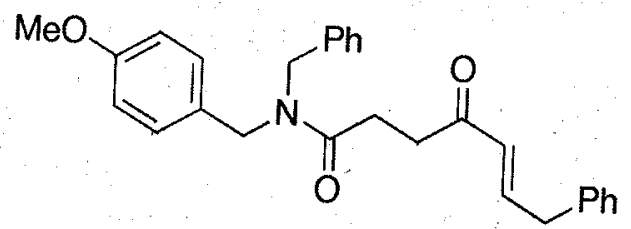

Relax. de lay $0.200 \mathrm{sec}$

Pulse 79.4 degrees

Acq. $t$ time 2.048
Width $8000.0 \mathrm{~Hz}$

16 repetitions

OBSERVE H1, 499.6156790 MHZ

Line broadening $0.1 \mathrm{~Hz}$

Fotal time $0 \mathrm{~min}, 40 \mathrm{sec}$

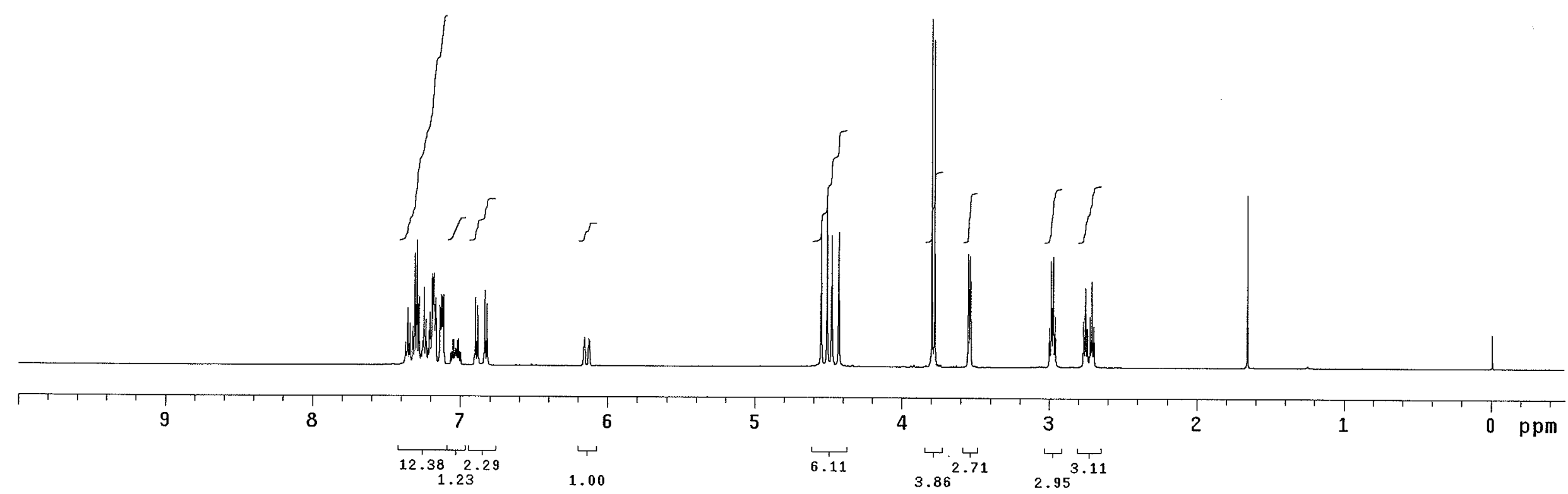




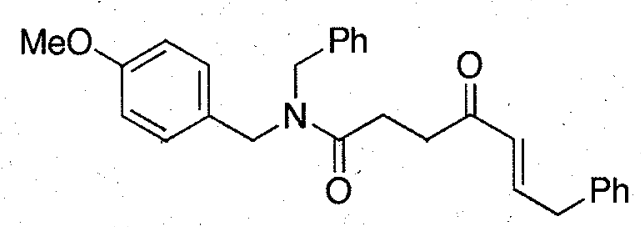

File: $6810-2-130$

Pulse sequence: s2pu

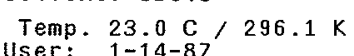

Pulse 40.0 degrees

width $29996.3 \mathrm{~Hz}$

OBSERVE C13,

DECOUPLE H1, 499.6181772 MHZ

Power $34 \mathrm{~dB}$

CAL $2-16$ sy on

DATA PROCESSING

Line broadening $1.0 \mathrm{~Hz}$

Fize 65536 hr, 55 m

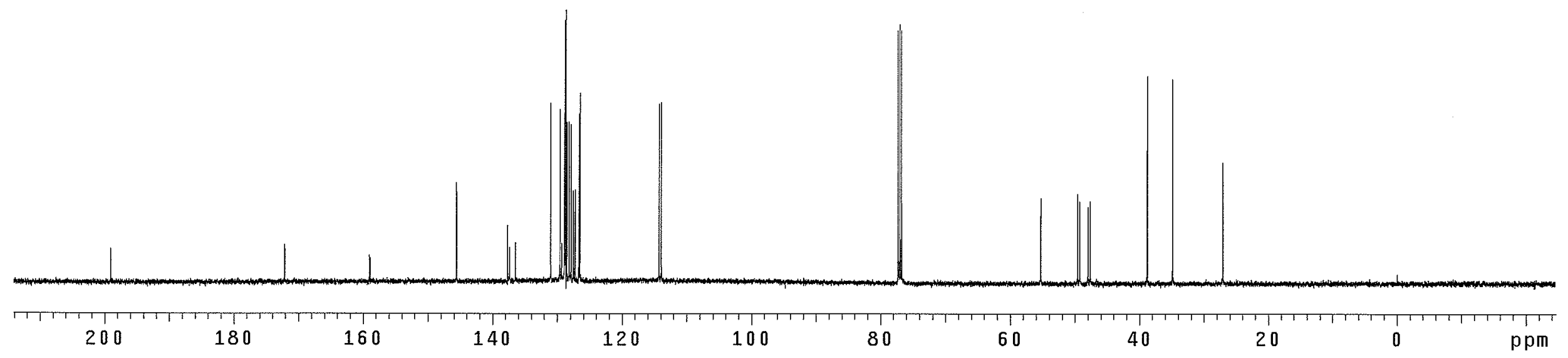




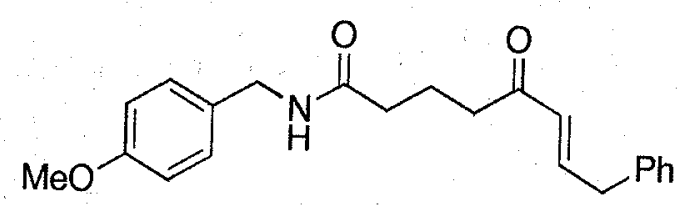

Re lax. de lay $0.200 \mathrm{sec}$

Pulse 79.4 degrees

width $8000.0 \mathrm{~Hz}$

16 repetitions

OBSERVE H1, 499.6156713 MHZ

Line broadening $0.1 \mathrm{~Hz}$

Fotal time $0 \mathrm{~min}, 40 \mathrm{sec}$

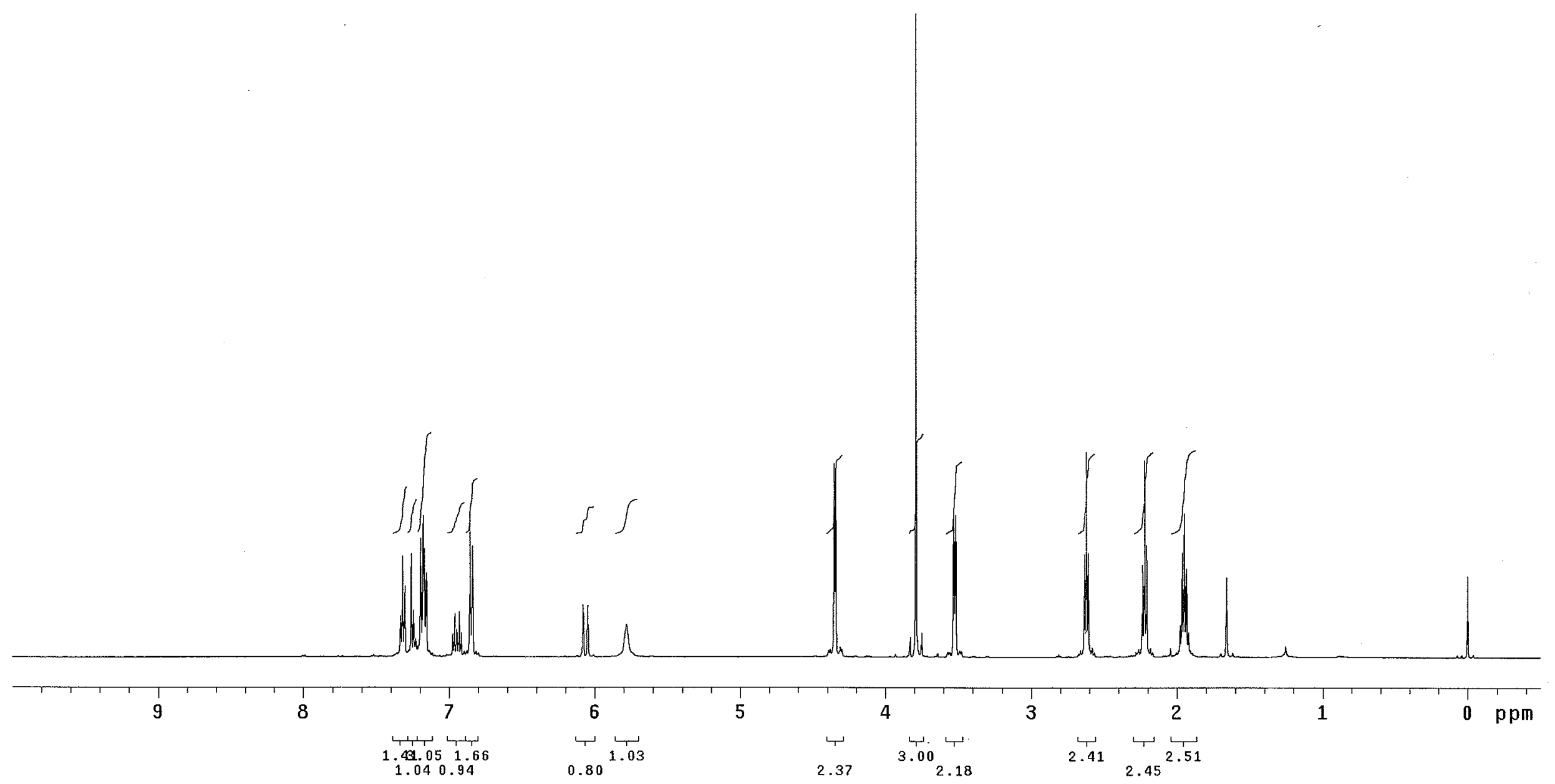




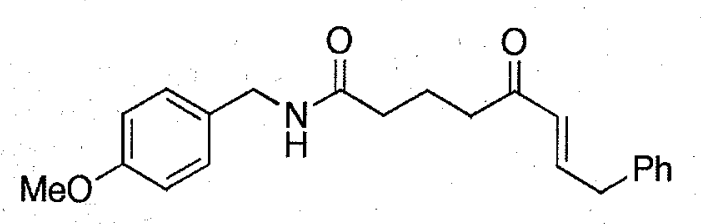

Puise 40.0 degrees

Acq time $1.092 \mathrm{sec}$

4560 repetitions

OBSERVE C13, $125.6284698 \mathrm{MHZ}$

DECOUPLE H1,

cont inuous ly on

WALTZ-16 modulat

DA A pocessina $1.0 \mathrm{~Hz}$

FT size 65536

Total time $30 \mathrm{hr}, 35 \mathrm{~min}, 30 \mathrm{sec}$

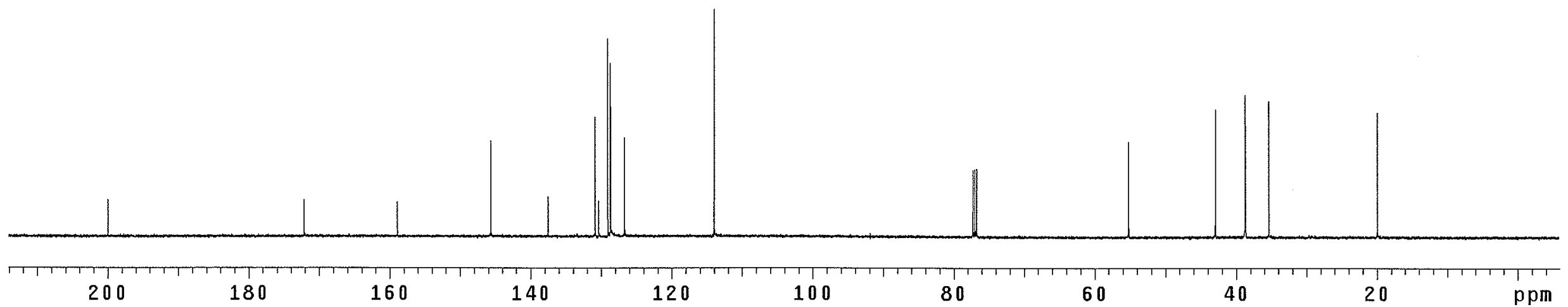




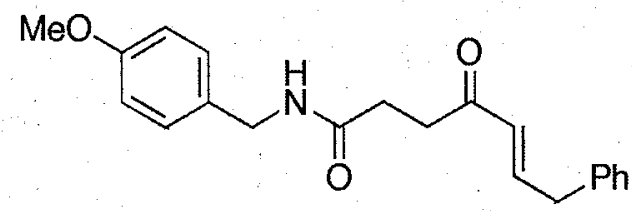

Re lax. de Tay $0.200 \mathrm{sec}$

Pulse 79.4 degrees
Acq. time $2.048 \mathrm{sec}$

Width $8000.0 \mathrm{~Hz}$

OBSERVE HI 499.6156718 MHZ

DATA PROCESSING
Line broadening $0.1 \mathrm{~Hz}$

Line broadening $0.1 \mathrm{~Hz}$
FT size $32768 \mathrm{~min}, 40 \mathrm{sec}$
Total time $0 \mathrm{~min}$

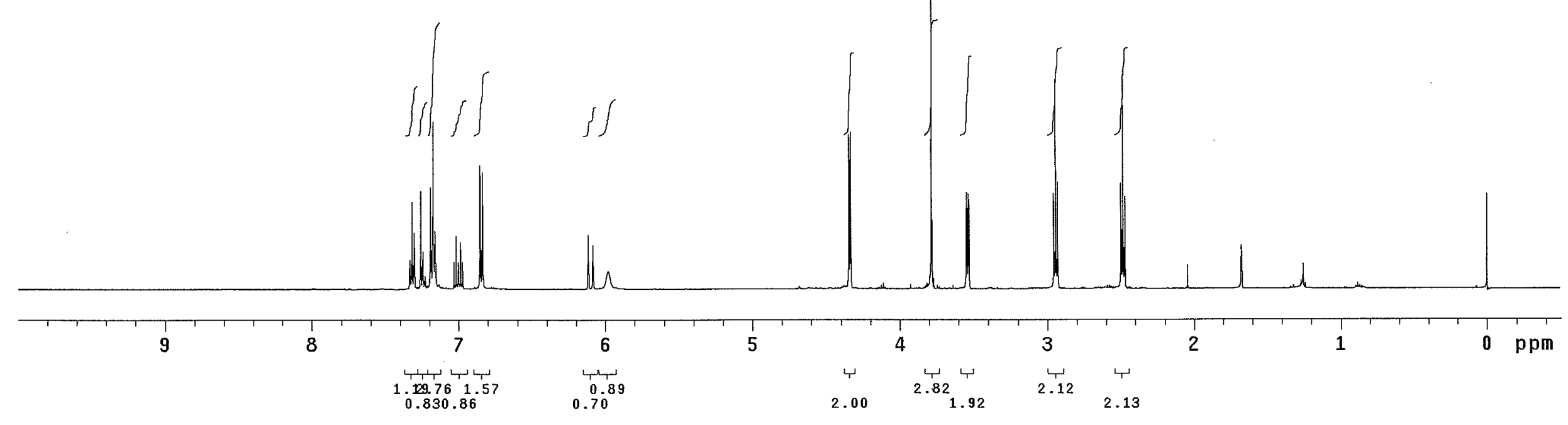




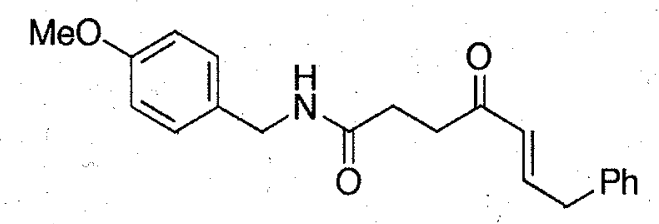

Pulse 40.0 degrees

Acq. time 1.092 sec

3682 repetitions

OBSERVE C13, $125.6284653 \mathrm{MHZ}$

DECOUPLE H1, 499.6181772 MHZ

cont inuously on

WALTZ-16 modulated

Line broadening $1.0 \mathrm{~Hz}$

FT size 65536

Total time $30 \mathrm{hr}, 35 \mathrm{~min}, 30 \mathrm{sec}$

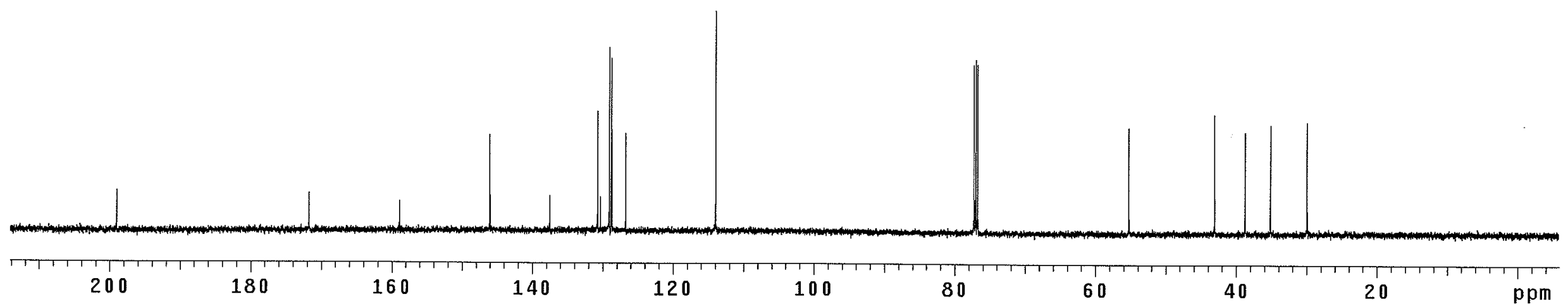




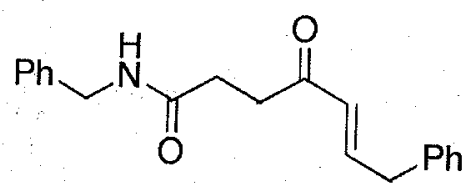

INOVA-500 "Mnovasuo"

Re lax. de lay $0.200 \mathrm{sec}$
Pulse 79.4 degrees

Acq t time $2.048 \mathrm{se}$

width $8000.0 \mathrm{~Hz}$

OBSERVE H1 499.6156718 MHz

DATA PROCESSÍNG

Line broadening $0.1 \mathrm{~Hz}$

FT size 32768
Total time $0 \mathrm{~min}, 40 \mathrm{sec}$

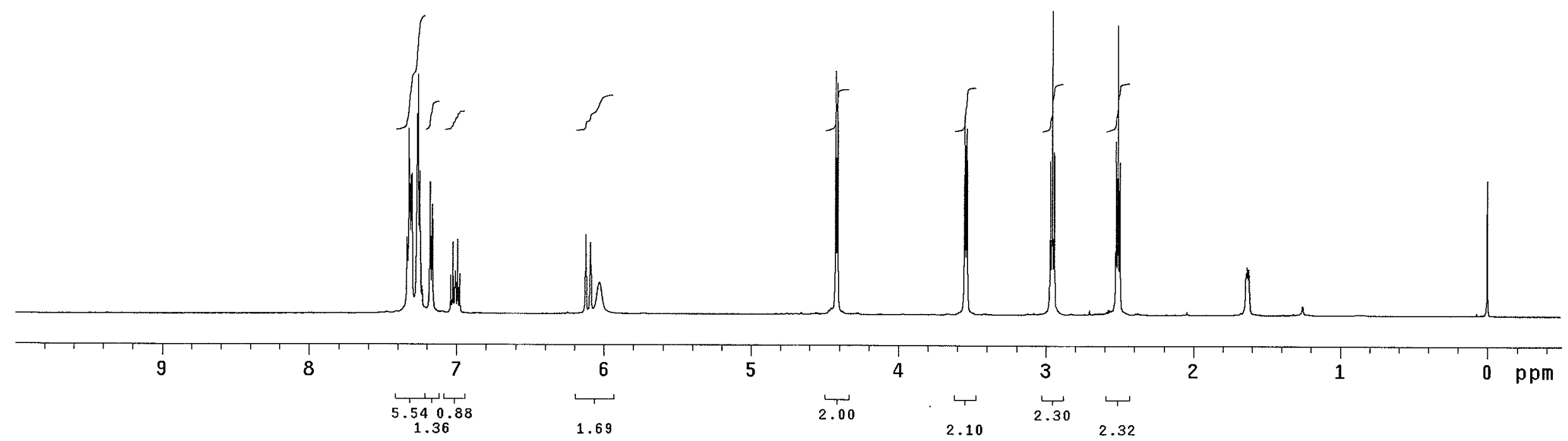




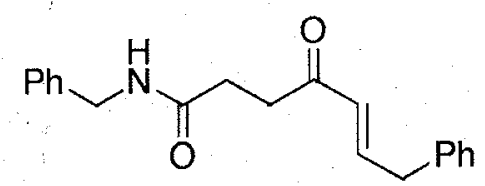

User: $1-14-87$
INOVA-500 "i nova500"

Pulse 40.0 degrees

Acq. time $1.092 \mathrm{sec}$

Width $29996.3 \mathrm{~Hz}$

OBSERVE C13, $125.6284653 \mathrm{MHZ}$

Power 34 aB

cont inuousiy on

DATA PROCESSING

Line broadening $1.0 \mathrm{~Hz}$

FT size 65536
Total time $30 \mathrm{hr}, 35 \mathrm{~min}, 30 \mathrm{sec}$

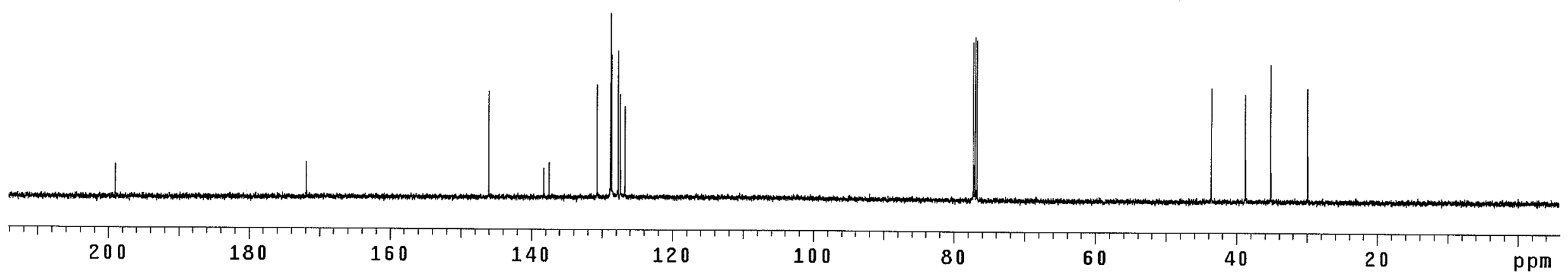




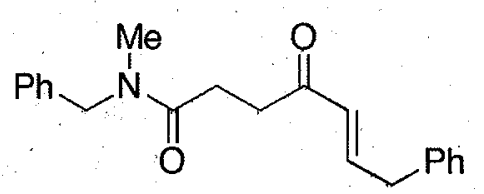

Relax. delay $0.200 \mathrm{sec}$

Pulse 79.4 degrees

Acq. time 2.048 sec

16 repetitions

OBSERVE H1, 499.6156728 MH

Line broadening $0.1 \mathrm{~Hz}$

FT size 32768
Total time $0 \mathrm{~min}, 40 \mathrm{sec}$

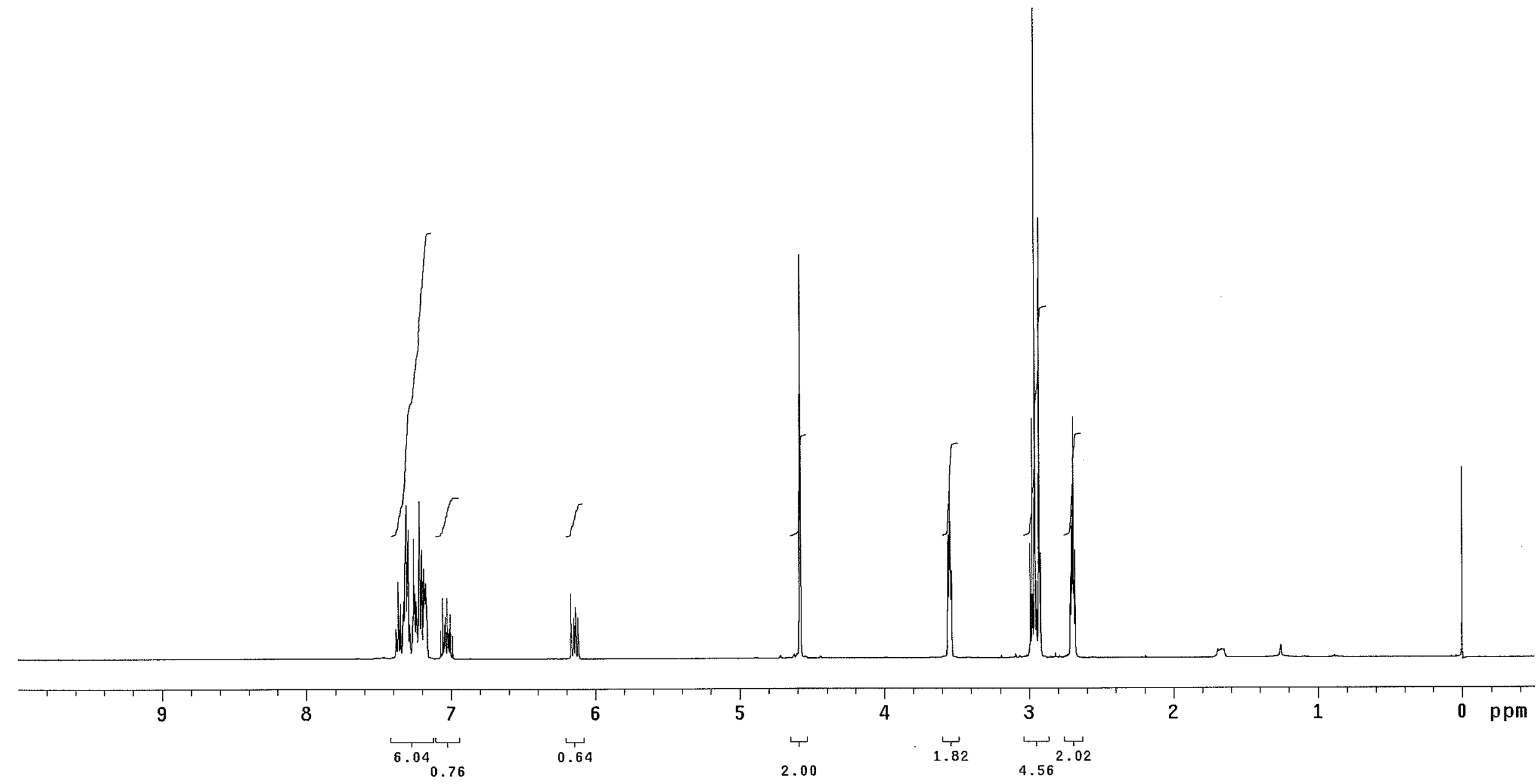




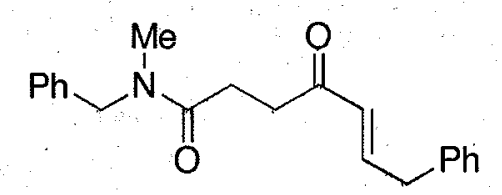

INOVA-500 " " i nova 500 "

Pulse 40.0 degrees
Acq. time 1.092 sec

Acq time $1.092 \mathrm{sec}$

4524 repetitions

OBSERVE C13, $125.6284653 \mathrm{MHz}$

Power $34 \mathrm{~dB}$,

WALTZ-16 ly on

DATA PROCESSING

Line broadening $1.0 \mathrm{~Hz}$

FT size 65536
Total time $3059 \mathrm{hr}, 11 \mathrm{~min}, 32 \mathrm{sec}$

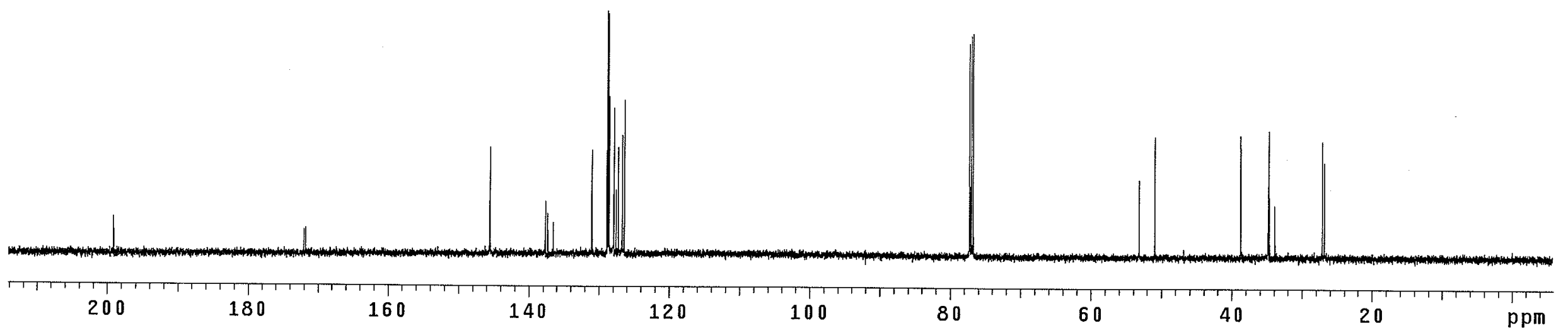

